\title{
Essential instabilities of fronts: bifurcation, and bifurcation failure
}

\author{
BJÖRN SANDSTEDE* and ARND SCHEEL $\uparrow$
}

\author{
* Department of Mathematics, Ohio State University, 231 West 18th \\ Avenue, Columbus, OH 43210, USA \\ $\dagger$ Institut für Mathematik I, Freie Universität Berlin, Arnimallee 2-6, \\ 14195 Berlin, Germany
}

Abstract. Various instability mechanisms of fronts in reaction-diffusion systems are analysed; the emphasis is on instabilities that have the potential to create modulated (i.e. time-periodic) waves near the primary front. Hopf bifurcations caused by point spectrum with associated localized eigenfunctions provide an example of such an instability. A different kind of instability occurs if one of the asymptotic rest states destabilizes: these instabilities are caused by essential spectrum. It is demonstrated that, if the rest state ahead of the front destabilizes, then modulated fronts are created that connect the rest state behind the front with small spatially periodic patterns ahead of the front. These modulated fronts are stable provided the spatially periodic patterns are stable. If, on the other hand, the rest state behind the front destabilizes, then modulated fronts that leave a spatially periodic pattern behind do not exist.

Received 26 July 2000

\section{Introduction}

Fronts describe the competition between two different states of a spatially extended system. In dissipative partial differential equations (PDEs) posed on the real line $x \in \mathbb{R}$, fronts occur frequently in situations where the equation admits two different spatially homogeneous states, say $U_{-}$and $U_{+}$. A front $Q(t, x)$ is a particular solution of the underlying PDE that preserves its shape while propagating with constant velocity towards $x=+\infty$, say. In other words, we have $Q(t, x)=Q\left(x-c_{*} t\right)$ where $c_{*}>0$ is the speed of propagation, and $Q(\xi) \rightarrow U_{ \pm}$as $\xi \rightarrow \pm \infty$, see figure 1. The direction of propagation singles out a preferred state of the system; in figure 1, the preferred state is $U_{-}$since the front travels to the right. Besides mere existence of fronts, a natural question is as to their dynamical stability properties. Only stable fronts describe the competition between the two rest states accurately for an open set of initial data.

Arguably, the most simple example is the Nagumo equation 


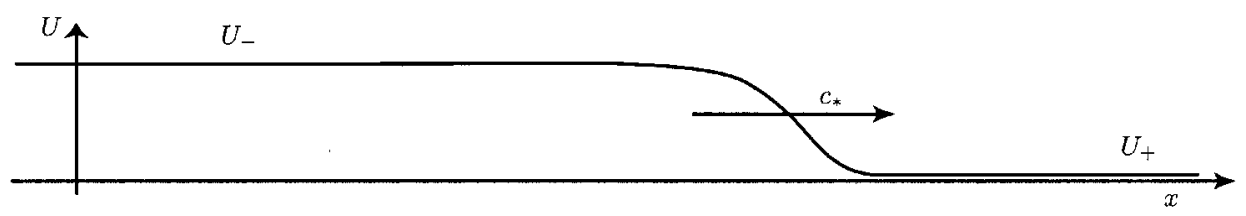

Figure 1. A schematic picture of a front.

$$
\partial_{t} U=\partial_{x x} U+U(1-U)(U-a), \quad x \in \mathbb{R}
$$

for scalar functions $U \in \mathbb{R}$. For every fixed $a \in(0,1 / 2)$, there exists a unique, up to spatial translates, front that connects the stable state $U_{-}=1$ to the stable state $U_{+}=0$. This front is monotone, and its wave speed $c_{*}$ is strictly positive. Any solution with initial data $U_{0}(x)$ that satisfies $U_{0}(x) \in(0,1)$ for $x \in \mathbb{R}$ with $U_{0}(x) \rightarrow 0$ as $x \rightarrow-\infty$ and $U_{0}(x) \rightarrow 1$ as $x \rightarrow+\infty$ converges to the aforementioned front or an appropriate spatial translate thereof.

Another example is the Gray-Scott model

$$
\begin{aligned}
& \partial_{t} U_{1}=d_{1} \partial_{x x} U_{1}-U_{1} U_{2}^{2}+F\left(1-U_{1}\right), \\
& \partial_{t} U_{2}=d_{2} \partial_{x x} U_{2}+U_{1} U_{2}^{2}-(F+k) U_{1} .
\end{aligned}
$$

In the parameter regime where $\Delta=1-4(F+k)^{2} / F$ is positive, there are three different homogeneous steady states. Two of them are stable with respect to the reaction kinetics: they are often referred to as the blue and the red state given by

$$
U_{-}=\left(\frac{1}{2}(1-\sqrt{\Delta}), \frac{F}{2(F+k)}(1+\sqrt{\Delta})\right), \quad U_{+}=(1,0) .
$$

respectively. Numerical simulations (Mazin et al. 1996) indicate that stable fronts that connect these two rest states exist for a wide range of parameters in the bistable regime. An explicit expression of the front for one particular set of parameters $\left(d_{1}, d_{2}, k, F\right)$ has been given by Hale et al. $(1999,2000)$. Analytical existence or stability results for fronts in the Gray-Scott model are not known, at least to our knowledge.

More generally, we concentrate on reaction-diffusion systems

$$
U_{t}=D U_{x x}+F(U ; \mu), \quad x \in \mathbb{R}
$$

with species $U=\left(U_{1}, \ldots, U_{N}\right) \in \mathbb{R}^{N}$ and a one-dimensional control parameter $\mu \in \mathbb{R}$. The diffusion matrix $D=\operatorname{diag}\left(d_{1}, \ldots, d_{N}\right)$ is diagonal with strictly positive entries $d_{j}>0$. We assume the existence of a front $Q\left(x-c_{*} t\right)$ with $Q(\xi) \rightarrow U_{ \pm}$for $\xi \rightarrow \pm \infty$. We also assume that the wave speed $c_{*}$ of the front is not equal to zero. Without loss of generality, we can then assume that the wave speed $c_{*}>0$ is positive; otherwise, replace $x$ by $-x$. We analyse equation (1) in a co-moving frame $\xi=x-c_{*} t$

$$
U_{t}=D U_{\xi \xi}+c_{*} U_{\xi}+F(U ; \mu), \quad \xi \in \mathbb{R},
$$

and investigate its linearization about $Q(\xi)$ :

$$
L_{Q} V:=D \partial_{\xi \xi} V+c_{*} \partial_{\xi} V+\partial_{U} F(Q(\xi) ; \mu) V, \quad \xi \in \mathbb{R} .
$$


Stability is determined by the spectrum of $L_{Q}$. Note that $\lambda=0$ is always an eigenvalue of $L_{Q}$ with eigenfunction $Q_{\xi}(\xi)$ due to translation invariance of equation (2).

In this article, we shall address the following two issues: 'How can fronts destabilize, and what patterns are created at the onset to instability?' We focus on Hopf bifurcations where $L_{Q}-\mathrm{i} \omega_{*}$ is not invertible for some $\omega_{*} \neq 0$. In such a situation, we expect that time-periodic waves are created that have a temporal frequency close to $\omega_{*}$. There are three different cases that we have to address.

First, suppose that $\lambda=\mathrm{i} \omega_{*}$ is an isolated eigenvalue of the linearization $L_{Q}$ about the front. The associated eigenmode then decays exponentially to zero as $\xi \rightarrow \pm \infty$, whence we call such an instability 'localized'. In this situation, we therefore encounter a classical Hopf bifurcation with an additional translation symmetry. The analysis is quite standard and essentially goes back to work by Pospiech (1992) and Henry (1981) who used Lyapunov-Schmidt reduction. For the sake of completeness, we give an alternative geometric description using the skew-product structure on the centre manifold (see Sandstede et al. 1997) that exploits the translation symmetry. Typically, a unique modulated front bifurcates that is time-periodic in an appropriate co-moving frame. Stability of the modulated front depends simply on the direction of branching. The created pattern is a time-periodic, spatially exponentially localized modulation of the primary front that manifests itself as a temporal oscillation of the formerly constant wave speed.

The remaining two cases occur if $\lambda=\mathrm{i} \omega_{*}$ is not isolated as an element of the spectrum of $L_{Q}$. We call such an instability 'essential' since it is caused by the essential spectrum crossing the imaginary axis. Since the essential spectrum is created by the asymptotic rest states $U_{ \pm}$, essential instabilities are not triggered by the front itself as in the first case considered above but by one of the asymptotic rest states $U_{+}$ or $U_{-}$. Thus, what distinguishes the two cases mentioned above is which rest state destabilizes: the rest state $U_{+}$ahead of the front or the rest state $U_{-}$behind the front. Before we outline our results, we recall what happens near the marginally stable rest state (without the front being present) near the onset to instability. In a co-moving frame, the three possible codimension-one instabilities of a homogeneous rest state that cause spectrum on the imaginary axis are Turing, Hopf and Turing-Hopf bifurcations. In all these cases, a continuum of temporally and spatially periodic small-amplitude patterns bifurcates from the homogeneous state. We refer to these patterns as 'basic patterns'. A Turing instability breaks only translation invariance: the bifurcating small basic patterns are spatially periodic with a sinusoidal shape and stationary with respect to time in the steady frame; they exist for any wavenumber $k$ close to the critical wavenumber $k_{*} \neq 0$. A Hopf bifurcation breaks only time invariance: the resulting basic patterns are time-periodic with frequency $\omega$ and spatially periodic with wavenumber $k \sim 0$; they exist for arbitrary temporal frequencies $\omega$ close to the critical frequency $\omega_{*} \neq 0$. In a Turing-Hopf bifurcation, waves travelling to the left and right as well as standing waves may be created. Both, temporal and spatial, invariance is broken by the critical mode; see figure 2.

In summary, we expect that, at the onset to an essential instability, small basic patterns are created near the rest state that triggers the instability. Next, we have to take the presence of the global front into account that connects the marginally unstable rest state with a different stable rest state. As mentioned before, we have to distinguish between the cases where the rest state $U_{+}$ahead of the front or the rest state $U_{-}$behind the front destabilizes; recall that we assumed that the wave speed $c_{*}$ of the front is positive so that the front always travels to the right towards $U_{+}$. 

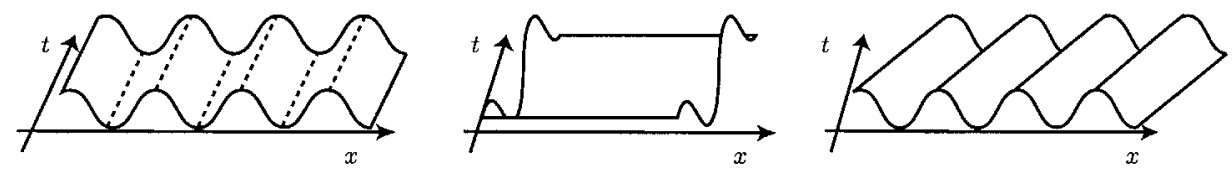

Figure 2. A schematic plot of the critical modes for Turing, Hopf, and TuringHopf instabilities.

First, assume that the rest state $U_{+}$becomes unstable. Thus, beyond the onset of instability, the primary front travels towards the unstable rest state $U_{+}$, leaving the stable rest state $U_{-}$behind. We demonstrate that modulated fronts bifurcate near the primary front. The modulated fronts connect the stable state $U_{-}$at $\xi=-\infty$ with one of the basic patterns near $U_{+}$at $\xi=+\infty$. The bifurcating modulated fronts are time-periodic in an appropriate moving frame. For any fixed value of the parameter $\mu$, taken along the direction of branching, there exists a continuum of modulated fronts parameterized by the wavelength of the asymptotic basic pattern. We prove that marginal stability of the primary front together with stability of the asymptotic basic pattern implies spectral stability of the bifurcating modulated front. We emphasize that there are many other modulated waves that bifurcate near an essential instability ahead of the front: in addition to the persisting primary front, there is a continuum of modulated fronts, for each given non-zero minimal temporal period, that connect the stable state $U_{-}$to the unstable state $U_{+}$; all these waves, however, are unstable. They can be thought of as interpolating between the primary front and the aforementioned modulated wave that connects to the basic pattern.

Second, assume that the rest state $U_{-}$destabilizes. Beyond the onset to instability, the primary front then travels towards the stable state $U_{+}$, leaving the unstable rest state $U_{-}$behind. In this situation, we prove that modulated fronts that connect one of the basic patterns at $\xi=-\infty$ with the rest state $U_{+}$at $\xi=+\infty$ cannot exist. In other words, the only modulated wave that exists nearby is the, then unstable, primary front itself.

Mathematically, the aforementioned three cases can be distinguished by Fredholm properties of the operator $L_{Q}-\mathrm{i} \omega_{*}$. Here, an operator $L: X \rightarrow Y$ is a Fredholm operator if the range $\mathrm{R}(L)$ of $L$ is closed in $Y$ and if both the dimension of the null space $\mathrm{N}(L)$ and the codimension of $\mathrm{R}(L)$ are finite. If $L$ is Fredholm, its index is defined as $i(L):=\operatorname{dim} \mathrm{N}(L)-\operatorname{codim} \mathrm{R}(L)$. Note that the sign of the index is related to the solvability properties of the equation $L x=y$ for given $y \in Y$ : roughly speaking, if the index $i(L)$ is zero, we expect that we can solve this equation uniquely for $x \in X$ for any given $y \in Y$; if $i(L)$ is negative, then the codimension of $R(L)$ is larger than the dimension of $\mathrm{N}(L)$, and we have more equations than variables so that we do not expect to have a solution; finally, if the index $i(L)$ is positive, then there are more variables than equations, and we expect to find many solutions. If $L$ is Fredholm, it is possible to use standard perturbation analysis to solve nonlinear equations of the form $L x=N(x)$; see for instance Golubitsky and Schaeffer (1985) for more details.

In the case of a Hopf instability induced by isolated eigenvalues $\pm \mathrm{i} \omega_{*}$, the operator $L_{Q}-\mathrm{i} \omega_{*}$ is a Fredholm operator with index zero. Standard perturbation theory, i.e. Lyapunov-Schmidt reduction, is then readily applicable. The last two cases mentioned above are characterized by the fact that the operator $L_{Q}-\mathrm{i} \omega_{*}$ is not a 
Fredholm operator; the range of this operator is not closed. As a consequence, classical perturbation theory fails. The above discussion of our main results shows that this is not just a purely mathematical artefact. If we had thought of an essential instability as a continuum of simultaneous Hopf bifurcations that occur separately for each eigenmode in the essential spectrum, then we would have expected that, for each fixed wavenumber, a unique modulated front bifurcates regardless of where the instability occurs, ahead or behind the front. Instead, many, and not just one, modulated fronts are created for instabilities ahead of the primary front, but none occur for instabilities behind the front. Thus, the predictions from a comparison with Hopf bifurcations are not correct. The behaviour of Fredholm indices, however, provides us with a clue as to what distinguishes Turing instabilities ahead and behind the front: for a Turing instability ahead of the front, the Fredholm index of $L_{Q}-\lambda$ changes from zero to one as $\lambda$ crosses from right to left through the essential spectrum. Thus, we expect an under-determined system of equations and therefore the existence of bifurcating solutions. On the other hand, for a Turing instability behind the front, the Fredholm index of $L_{Q}-\lambda$ changes from zero to minus one, and we expect an over-determined system of equations for which no bifurcating solutions exist.

Finally, we briefly outline our approach to analysing essential instabilities. The key is to take the spatial variable into account which plays a far more important role than the time variable. To illustrate our approach, suppose first that we seek the primary travelling front itself. In an appropriate co-moving frame, the front is then a stationary solution to the reaction-diffusion system

$$
U_{t}=D U_{\xi \xi}+c_{*} U_{\xi}+F(U ; \mu), \quad \xi \in \mathbb{R} .
$$

In particular, the front satisfies the ordinary differential equation (ODE)

$$
D U_{\xi \xi}+c_{*} U_{\xi}+F(U ; \mu)=0
$$

or, written as a first-order system

$$
\left(\begin{array}{c}
U_{\xi} \\
V_{\xi}
\end{array}\right)=\left(\begin{array}{c}
V \\
-D^{-1}\left(c_{*} V+F(U ; \mu)\right)
\end{array}\right) .
$$

We can then use dynamical-system methods to seek fronts as heteroclinic orbits that connect the equilibria $\left(U_{-}, 0\right)$ and $\left(U_{+}, 0\right)$ with each other. To find modulated fronts, we adopt the very same approach: we anticipate the temporal period of the modulated waves that we seek, and consider the reaction-diffusion system as a dynamical system in the spatial variable. In other words, we consider the equation

$$
\left(\begin{array}{c}
U_{\xi} \\
V_{\xi}
\end{array}\right)=\left(\begin{array}{c}
V \\
-D^{-1}\left(-U_{t}+c_{*} V+F(U ; \mu)\right)
\end{array}\right)
$$

where $(U, V)$ are time-periodic functions for each fixed $\xi$, and seek modulated fronts as heteroclinic orbits. The main technical obstacle to implementing these ideas is that the aforementioned equation is not a dynamical system as the initial-value problem is ill-posed. We have, however, demonstrated how this obstacle can be resolved by using exponential dichotomies (see Peterhof et al. (1997), Sandstede and Scheel (1999a, 2000)). 
The plan of this article is as follows. In section 2, we classify the linear stability problem. Sections 3 and 4 contain the main bifurcation results in the case of localized and essential instabilities, respectively. Sections 5 and 6 are devoted to the proof of the main results, namely existence, non-existence, and stability of bifurcating modulated fronts. Afterwards, we discuss two applications: first an artificial model problem that satisfies all assumptions of our theorems, and second, the Gray-Scott model for which we have verified the assumptions numerically. We conclude with an extensive discussion in section 9 .

\section{Spectra of fronts}

We begin by reviewing results on the spectra of fronts. The linearization about the front $Q$ is given by

$$
L_{Q} V=D V_{\xi \xi}+c_{*} V_{\xi}+\partial_{U} F(Q(\xi) ; \mu) V
$$

with $Q(\xi) \rightarrow U_{ \pm}$as $\xi \rightarrow \pm \infty$. We consider $L_{Q}$ as an operator defined on the space $\mathcal{L}^{2}\left(\mathbb{R}, \mathbb{R}^{N}\right)$. The function spaces $\mathcal{C}^{0}$ or $\mathcal{L}^{p}$ give the same spectra. We say that $\lambda$ is in the point spectrum of $L_{Q}$ if $L_{Q}-\lambda$ is a Fredholm operator with index zero so that the null space $\mathrm{N}\left(L_{Q}-\lambda\right)$ is non-trivial. If $L_{Q}-\lambda$ is either not Fredholm or else its index is non-zero, then we say that $\lambda$ is in the essential spectrum. Palmer (1988) has characterized the spectrum in terms of the associated ODE

$$
\begin{aligned}
V_{\xi} & =W \\
W_{\xi} & =D^{-1}\left(-c_{*} W-\partial_{U} F(Q(\xi) ; \mu) V+\lambda V\right)
\end{aligned}
$$

which we also write as

$$
\left(\begin{array}{c}
V_{\xi} \\
W_{\xi}
\end{array}\right)=A(\xi ; \lambda)\left(\begin{array}{c}
V \\
W
\end{array}\right)
$$

where $A(\xi ; \lambda) \rightarrow A_{ \pm}(\lambda)$ for $\xi \rightarrow \pm \infty$; see also Henry (1981). Palmer proved that $L_{Q}-\lambda$ is Fredholm if and only if both, $A_{+}(\lambda)$ and $A_{-}(\lambda)$, are hyperbolic, i.e. if neither matrix has a spectrum on the imaginary axis. The Fredholm index $i(\lambda)$ of $L_{Q}$ is then given by

$$
i(\lambda)=i_{-}(\lambda)-i_{+}(\lambda)
$$

where $i \pm(\lambda)$ are the dimensions of the generalized unstable eigenspaces of $A_{ \pm}(\lambda)$, i.e. the dimensions of the eigenspaces associated with eigenvalues of $A_{ \pm}(\lambda)$ that have positive real part. In particular, the Fredholm index changes whenever eigenvalues of $A_{+}(\lambda)$ or $A_{-}(\lambda)$ cross the imaginary axis. The aforementioned results can be generalized to modulated waves, where $Q=Q(t, \xi)$ is time-periodic; see section 6 below.

As an immediate consequence of the above characterization, there are three different typical mechanisms that lead to an unstable front:

(I) point spectrum of $L_{Q}$ crosses the imaginary axis, while the essential spectrum of $L_{Q}$ is uniformly confined to the open left half-plane;

(II) essential spectrum associated with $U_{+}$crosses the imaginary axis;

(III) essential spectrum associated with $U_{-}$crosses the imaginary axis. 
Note that, in the first case, point spectrum always corresponds to isolated eigenvalues with finite multiplicity since we are to the right of the essential spectrum, a region which cannot consist entirely of point spectrum.

In addition to the critical spectrum that crosses the imaginary axis, there is always at least one additional eigenvalue on the imaginary axis, namely $\lambda=0$ with eigenvector $Q_{\xi}(\xi)$ induced by translation symmetry.

We conclude this section by classifying instability mechanisms for the asymptotic spatially homogeneous rest states. We discuss these instabilities in the steady frame $x$ where reflection symmetry imposes restrictions on what bifurcations arise typically. Fourier transform (or a reference to Palmer's results above) allows us to express critical spectrum in an algebraic dispersion relation. We write $\nu(\lambda ; \mu)$ for the critical eigenvalue, of either $A_{+}(\lambda ; \mu)$ or $A_{-}(\lambda ; \mu)$ depending of which rest state's essential spectrum destabilizes, that is close to the imaginary axis so that $\nu\left(\mathrm{i} \omega_{*} ; 0\right)=\mathrm{i} k_{*}$. The four possible cases are:

(i) $\omega_{*}=0, k_{*}=0$, typically corresponding to a fold in the kinetics;

(ii) $\omega_{*}=0, k_{*} \neq 0$, the Turing instability;

(iii) $\omega_{*} \neq 0, k_{*}=0$, a Hopf instability in the kinetics;

(iv) $\omega_{*} \neq 0, k_{*} \neq 0$, the Turing-Hopf instability.

We do not discuss case (i). Plots of the critical modes for cases (ii)-(iv) can be found in figure 2 in section 1 .

\section{Localized instabilities: Hopf bifurcations with translation symmetry}

We briefly discuss case (I) where point spectrum crosses the imaginary axis at $\lambda= \pm \mathrm{i} \omega_{*}$.

Hypothesis 1 (local Hopf bifurcation). For $\mu=0$, the essential spectrum of $L_{Q}$ is contained in the open left half-plane. Moreover, $\operatorname{spec}\left(L_{Q}\right) \cap \mathrm{i} \mathbb{R}=\left\{0, \pm \mathrm{i} \omega_{*}\right\}$ for some $\omega_{*} \neq 0$, and these eigenvalues have algebraic multiplicity one. The eigenvalues $\pm \mathrm{i} \omega_{*}$ cross the imaginary axis transversely as $\mu$ crosses through zero.

We formulate the next theorem for the space $\mathcal{C}_{\text {unif }}^{0}$ that consists of bounded, uniformly continuous functions.

Theorem 1 (centre manifolds and skew-product structure; Henry 1981, Sandstede et al. 1997). Assume that Hypothesis 1 is met, then there exists a three-dimensional centre manifold for equation (2) in $\mathcal{C}_{\text {unif }}^{0}$ that contains the front. The centre manifold is diffeomorphic to $\Sigma=\mathbb{R} \times\{z \in \mathbb{C} ;|z| \leq 1\}$, and the $P D E$ (2), restricted to the centre manifold and written in the coordinates $(\tau, z) \in \Sigma$, is of skew-product form:

$$
\dot{\tau}=\tau g(z, \bar{z} ; \mu), \quad \dot{z}=h(z, \bar{z} ; \mu)
$$

with $g(0,0 ; 0)=c_{*}$ and $h(z, \bar{z} ; \mu)=\mathrm{i} \omega_{*} z+\mu a_{1} z+a_{2} z|z|^{2}+\mathrm{O}\left(|z|^{4}+|\mu|^{2} z\right)$ where $\operatorname{Re} a_{1} \neq 0$. The set $\{(\tau, 0) ; \tau \in \mathbb{R}\}$ parameterizes the translates of the primary front.

Corollary 1 (bifurcations to modulated waves; Pospiech 1992). Assume that, in addition to the assumptions of Theorem 1 , we have $\operatorname{Re} a_{2} \neq 0$; see Theorem 1 . For every $\mu$ with $\mu$ close to zero and $\operatorname{sign}(\mu)=-\operatorname{sign}\left(\operatorname{Re} a_{1}\right) \operatorname{sign}\left(\operatorname{Re} a_{2}\right)$, there exists a unique (up to spatial and temporal translations) modulated front $Q_{\mu}(t, x-c(\mu) t)$ so that $Q_{\mu}(t+p(\mu), \cdot)=Q_{\mu}(t, \cdot)$ where $c(\mu)$ and $p(\mu)$ are continuous functions with $c(0)=c_{*}$ and $p(0)=2 \pi / \omega_{*}$. If $\operatorname{Re} a_{2}<0$, then the set consisting of all spatial and 


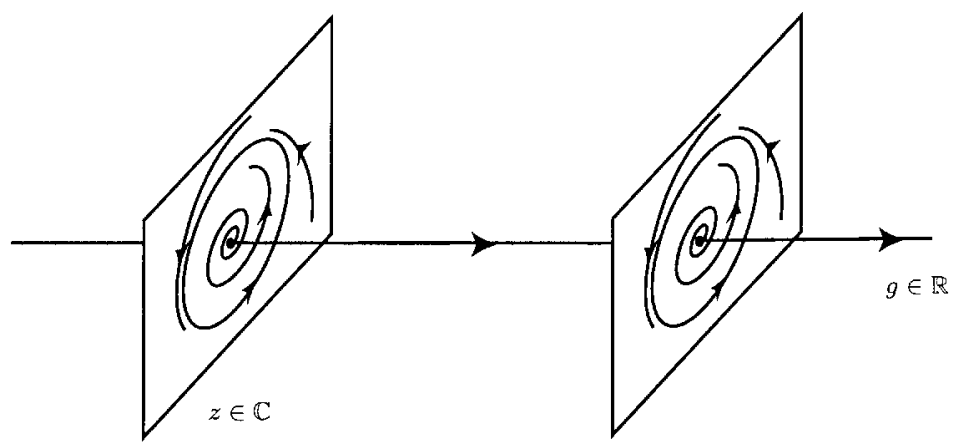

Figure 3. The centre manifold with the bifurcating stable periodic orbit in the $z$-component.

temporal translates of the modulated front is exponentially attracting with asymptotic phase.

We refer to figure 3 for a schematic picture of the skew-product dynamics after bifurcation.

The interpretation of the result stated in the corollary is as follows. Since the matrices $A_{ \pm}\left(\mathrm{i} \omega_{*}\right)$ are both hyperbolic, the Hopf eigenmodes are exponentially localized. Periodic oscillations in the $z$-equation describe exponentially localized variations of the shape of the front interface. They induce, via $g(z, \bar{z} ; \mu)$, a periodic modulation of the speed of propagation of the modulated front.

An example of a specific reaction-diffusion system where a front destabilizes in a local Hopf bifurcation has been studied in Pospiech (1992, Theorem 5.24).

\section{Essential instabilities: Turing instabilities ahead or behind the front}

This section contains our main results. We focus on the case where the rest states undergo a Turing bifurcation; see case (ii) in section 2. Similar results are true in cases (iii) and (iv) of Hopf and Turing-Hopf instabilities; they are discussed in section 9 . We begin by stating the genericity assumptions that we shall need below.

Hypothesis 2 (front). We assume that $Q(\xi)$ is a travelling front so that, for some $c_{*}>0$,

$$
D Q_{\xi \xi}+c_{*} Q_{\xi}+F(Q ; 0)=0
$$

and $Q(\xi) \rightarrow U_{ \pm}$for $\xi \rightarrow \pm \infty$.

Hypothesis 3 (Turing bifurcation ahead of the front). We assume that the homogeneous rest state $U_{+}$undergoes a non-degenerate Turing instability with critical wave number $k_{*} \neq 0$, while $U_{-}$remains asymptotically stable. More precisely, we require that, for some $k_{*} \neq 0$ :

(i) $\operatorname{det}\left(-D k^{2}+\partial_{U} F\left(U_{-} ; 0\right)-\lambda\right) \neq 0$ for all $\lambda$ with $\operatorname{Re} \lambda \geq 0$ and all $k \in \mathbb{R}$;

(ii) $\operatorname{det}\left(-D k^{2}+\partial_{U} F\left(U_{+} ; 0\right)-\lambda\right) \neq 0$ for all $\lambda \neq 0$ with $\operatorname{Re} \lambda \geq 0$ and all $k \in \mathbb{R}$;

(iii) $\operatorname{det}\left(-D k^{2}+\partial_{U} F\left(U_{+} ; 0\right)\right) \neq 0$ for $|k| \neq k_{*}$;

(iv) there are constants $C_{1}, C_{k}$ and $C_{\mu}$ with $C_{1} \neq 0$ and $C_{k}, C_{\mu}>0$ such that 


$$
\begin{aligned}
\operatorname{det}\left(-D k^{2}+\partial_{U} F\left(U_{-} ; 0\right)-\lambda\right)= & C_{1}\left(\lambda-C_{k}\left(k-k_{*}\right)^{2}+C_{\mu} \mu\right) \\
& +\mathrm{O}\left(\mu^{2}+\left|k-k_{*}\right||\mu|+|\lambda|^{2}+\left(|\mu|+\left|k-k_{*}\right|+|\lambda|\right)^{3}\right) ;
\end{aligned}
$$

see also figure 5.

Hypothesis 4 (Turing bifurcation behind the front). We assume that the homogeneous rest state $U_{-}$undergoes a non-degenerate Turing instability with critical wave number $k_{*} \neq 0$, while $U_{+}$remains asymptotically stable. The precise assumptions are as in hypothesis 3 with $U_{+}$and $U_{-}$interchanged.

Hypothesis 5 (supercritical Turing bifurcation). We assume that the Turing bifurcation is supercritical: upon restricting the reaction-diffusion system to the space of even $2 \pi / k_{*}$-periodic functions, the resulting pitchfork bifurcation is supercritical so that the bifurcating Turing patterns are stable within this class of functions.

The criterion for supercriticality is equivalent to a negative sign of the coefficient $\gamma_{2}$ of the cubic term in the Ginzburg-Landau modulation equation

$$
A_{t}=A_{x x}+\gamma_{1} A+\gamma_{2} A|A|^{2}
$$

that governs the Turing instability.

Finally, we have to exclude additional spectrum of the front on the imaginary axis besides the spectrum that is necessarily induced by the Turing instability and by the translation symmetry. To state this hypothesis, we consider the operator $L_{Q}$, which represents the linearization about the front in a co-moving frame, in the exponentially weighted $\mathcal{L}^{2}$-spaces $\mathcal{L}_{\eta}^{2}$ with norm

$$
\|U\|_{\mathcal{L}_{\eta}^{2}}:=\left\|\mathrm{e}^{\eta \xi} U(\xi)\right\|_{\mathcal{L}^{2}}
$$

Hypothesis 6 (stability in weighted spaces). We assume that there is a constant $\eta_{*}>0$ such that, for all $0<\eta<\eta_{*}$, we have

$$
\operatorname{spec}_{\mathcal{L}_{\eta}^{2}}\left(L_{Q}\right) \cap\{\operatorname{Re} \lambda \geq 0\}=\{0\},
$$

and $\lambda=0$ is an eigenvalue in the point spectrum with algebraic multiplicity one.

Recall that we have $c_{*}>0$ so that the Turing patterns, which were stationary in the steady frame, travel to the left in a co-moving frame. The choice $\eta>0$ of the weight allows for patterns that travel to the left. This explains the absence of the essential spectrum on the imaginary axis in the weighted spaces.

Sandstede and Scheel (1999a) demonstrated that Hypothesis 6 is typically met for marginally stable pulses that are asymptotic to a rest state undergoing a Turing instability. The relevant hypothesis in Sandstede and Scheel (1999a) was stated in a slightly different way that made use of the geometric information from spatial dynamics that we explain later in section 5 .

First, we state a result on the existence and stability of small Turing patterns close to the rest state $U_{+}$(or $U_{-}$in the case where Hypothesis 4 is met). We illustrate these results in figure 4 .

Theorem 2 (existence and stability of Turing patterns). Assume that Hypotheses 5 and 3 (or 4) are met. There are functions $\mu_{\mathrm{ex}}(k)$ and $\mu_{\mathrm{st}}(k)$ defined for $k$ close to $k_{*}$ with expansions 


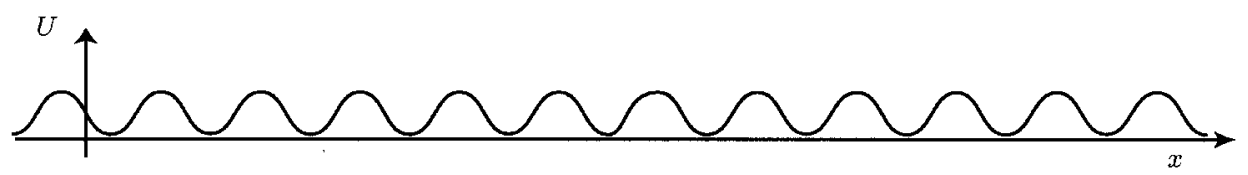

Figure 4. A stationary spatially periodic Turing pattern.

$$
\mu_{\mathrm{ex}}(k)=\frac{C_{k}}{C_{\mu}}\left(k-k_{*}\right)^{2}+\mathrm{O}\left(\left|k-k_{*}\right|^{3}\right)
$$

and

$$
\mu_{\mathrm{st}}(k)=3 \frac{C_{k}}{C_{\mu}}\left(k-k_{*}\right)^{2}+\mathrm{O}\left(\left|k-k_{*}\right|^{3}\right),
$$

where the constants $C_{k}, C_{\mu}$ are defined in Hypotheses 3 and 4, so that:

- if $\mu>\mu_{\mathrm{ex}}(k)$, then there exists a unique (up to translation) stationary $2 \pi / k$ periodic pattern $T_{\mu, k}(x)$ near $U_{+}$(or $U_{-}$);

- if $\mu>\mu_{\mathrm{st}}(k)>\mu_{\mathrm{ex}}(k)$, then the unique Turing pattern is spectrally stable.

In figure 5, we illustrate the typical shape of the dispersion relation near a Turing bifurcation as well as the existence and stability regions in a parameter-versuswavenumber plot.

The question addressed in the next two theorems is the existence of modulated fronts. If the Turing instability occurs at $U_{+}$, then we seek modulated fronts that connect the stable state $U_{-}$to the Turing patterns created at $U_{+}$. If, on the other hand, the Turing instability occurs at $U_{-}$, then stable modulated fronts should connect the Turing patterns created at $U_{-}$with the stable rest state $U_{+}$.

Theorem 3 (Turing instability ahead of the front). Assume that Hypotheses 2, 3, 5 and 6 are met. There are then constants $\delta, \tilde{\delta}>0$ such that, for any $\mu$ and $k$ with $|\mu|+\left|k-k_{*}\right|<\delta$ and $\mu>\mu_{\mathrm{ex}}(k)$, there exists a modulated front $Q_{\mu, k}\left(t, x-c_{\mu, k} t\right)$ with wave speed $c_{\mu, k}$ and temporal period $p_{\mu, k}=2 \pi /\left(c_{\mu, k} k\right)$, i.e. $Q_{\mu, k}\left(t+p_{\mu, k}, \cdot\right)=$ $Q_{\mu, k}(t, \cdot)$. The front is asymptotic to $U_{-}$at $\xi=-\infty$ and to the Turing patterns $T_{\mu, k}(x)$ at $\xi=+\infty$. More precisely, there are positive constants $C, \eta, \theta_{+}>0$ such that
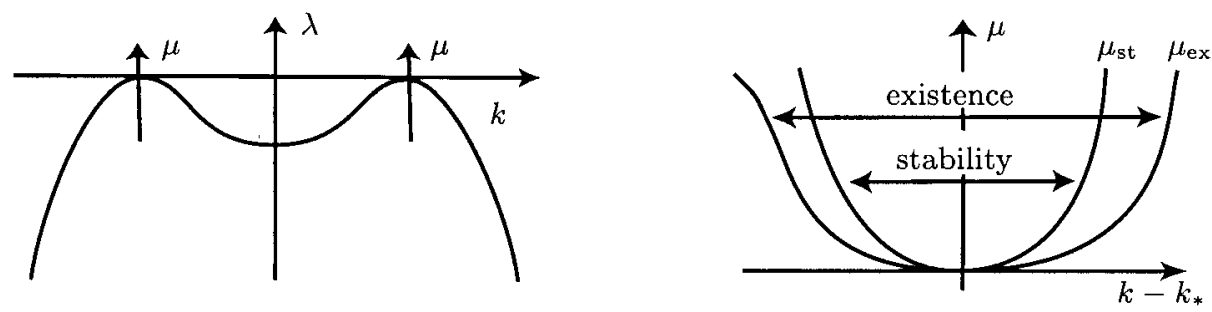

Figure 5. In the left figure, the eigenvalue $\lambda$ of the linearized operator $L$ is plotted over the wave number $k$. The curve is moving upwards with increasing $\mu$. In the right figure, the outer curve denotes the boundary of existence of Turing patterns $\mu_{\mathrm{ex}}(k)$, while the inner curve denotes the Eckhaus boundary $\mu_{\mathrm{st}}(k)$ of stability of the Turing patterns. 


$$
\begin{aligned}
\left|Q_{\mu, k}(t, \xi)-T_{\mu . k}\left(\xi+c_{\mu, k} t+\theta_{+}\right)\right| \leq C \mathrm{e}^{-\eta \xi} & \xi \geq 0, \\
\left|Q_{\mu, k}(t, \xi)-U_{-}\right| \leq C \mathrm{e}^{\eta \xi} & \xi \leq 0,
\end{aligned}
$$

uniformly in $t, k, \mu$. The front is unique in the class of time-periodic solutions in a frame moving with any speed $\tilde{c}$ with $\left|c_{*}-\tilde{c}\right|<\tilde{\delta}$ in a $(t, \xi)$-uniform $\delta$-neighbourhood of $Q(\xi)$, up to spatial and temporal translates.

If $\mu>\mu_{\mathrm{st}}(k)$ (see Theorem 2 for the definition of the Eckhaus boundary $\mu_{\mathrm{st}}(k)$ ), the modulated front is spectrally stable:

$$
\operatorname{spec}_{\mathcal{L}^{2}}\left(\Phi_{\mu, k}\right) \subset\{|\lambda| \leq 1\},
$$

where $\Phi_{\mu, k}$ denotes the linearization of the period map to the reaction-diffusion system evaluated at $Q_{\mu, k}$.

A schematic picture of the modulated front in the steady frame is shown in figure 6 . Thus, if the Turing instability occurs ahead of the front, then the stable stationary Turing patterns are invaded by the rest state $U_{-}$behind the front. The next result demonstrates that the case of a Turing instability behind the front is quite different; we also refer to figure 7 for a schematic snapshot of the dynamics.

Theorem 4 (Turing instability behind the front). Assume that Hypotheses 2, 4, 5 and 6 are satisfied. There is then a constant $\delta>0$ such that, for any $\mu, k$ and $\tilde{c}$ with $|\mu|+\left|k-k_{*}\right|+\left|\tilde{c}-c_{*}\right|<\delta$, there is no modulated front $Q_{\mu, k}(t, x-\tilde{c} t)$ with wave speed $\tilde{c}$ that is asymptotic to $U_{+}$for $\xi \rightarrow+\infty$

$$
\left|Q_{\mu, k}(t, \xi)-U_{+}\right| \rightarrow 0 \quad \text { as } \xi \rightarrow+\infty
$$

uniformly in $t$, and asymptotic to $T_{\mu, k}$ for $\xi \rightarrow-\infty$ so that

$$
\left|Q_{\mu, k}(t, \xi)-T_{\mu, k}\left(\xi+\tilde{c} t+\theta_{-}\right)\right| \rightarrow 0 \quad \text { as } \xi \rightarrow-\infty
$$

uniformly in $t$ for some $\theta_{-}$.

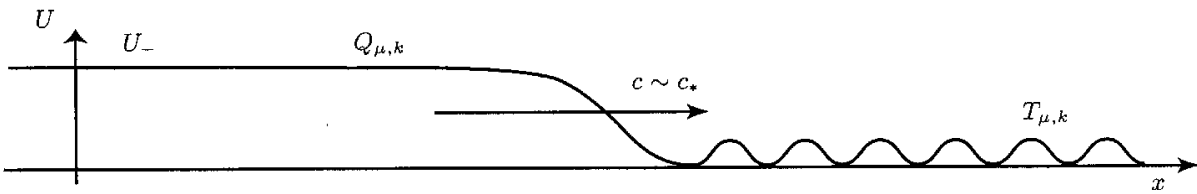

Figure 6. Turing bifurcation ahead of the front. The modulated front that invades a stable stationary Turing pattern, plotted in the steady frame.

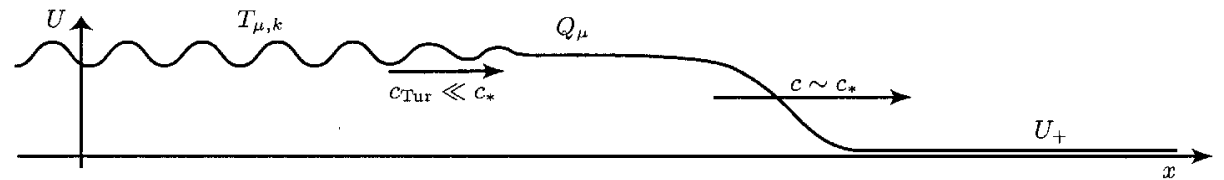

Figure 7. Turing bifurcation behind the front. The primary unstable front in the case where the Turing patterns are created behind the front, plotted in the steady frame. Instabilities behind the front eventually propagate with a smaller mean velocity than the front. Modulated fronts do not bifurcate in this situation. 
Existence and non-existence in the above theorems are proved in the next section. Stability of the modulated fronts in Theorem 3 is established in section 6 .

\section{Spatial dynamics on time-periodic functions}

In this section, we prove the statements concerning existence and non-existence in Theorems 3 and 4. As mentioned in section 1, the key idea is to regard the PDE as a dynamical system in the spatial variable, acting upon time-periodic functions. This approach had been used by Iooss et al. (1989) to investigate local instabilities in fluid problems and has been extended by Sandstede and Scheel (1999a) to global homoclinic bifurcations that correspond to Turing instabilities ahead and behind pulses. Sandstede and Scheel (1999a) analysed the linearized equation about the homoclinic orbit using exponential dichotomies for which more subtle robustness theorems are available. These robustness theorems replace the Fredholm properties that fail at the moment of bifurcation. In this section, we show how the arguments given in Sandstede and Scheel (1999a) can be adapted to global heteroclinic bifurcations. We refer to Sandstede and Scheel (1999a, 2000) for the technical details of the construction of invariant manifolds and focus instead on the geometric picture of the heteroclinic bifurcations.

As outlined in section 1, we begin by rewriting the reaction-diffusion system formally as a dynamical system in the spatial variable $\xi$, allowing for a periodic time-dependence of the solutions:

$$
u_{\xi}=v, \quad v_{\xi}=D^{-1}\left(u_{t}-c v-F(u ; \mu)\right)
$$

where $(u, v)(\xi, \cdot) \in Y=\mathcal{H}_{\text {per }}^{1 / 2}(0, p)^{N} \times \mathcal{L}_{\text {per }}^{2}(0, p)^{N}$ for each fixed $\xi \in \mathbb{R}$. We consider the temporal period $p$ as an additional free parameter. In the next step, we reformulate our assumptions in a dynamical-systems language for the $\xi$-dynamics.

First, the travelling-wave ODE is recovered upon restricting to the subspace $Y_{0}=\mathbb{R}^{2 M} \leq Y$ of time-independent functions. In fact, the PDE (2) is invariant under shifts of the time variable since it is autonomous. Considering the $\xi$-dynamics, this invariance becomes an equivariance of the dynamical system (3): the circle group $S^{1}$ acts on $Y$ via $(u, v)(t) \mapsto(u, v)(t-\theta)$ where $\theta \in S^{1}$. The fixed-point space of this $S^{1}$-action is then also dynamically invariant with respect to the $\xi$-evolution of equation (3). This fixed-point space is given by the subspace $Y_{0}$ of $Y$ that consists of all time-independent functions.

Equation (3) restricted to $Y_{0}$ reads

$$
u_{\xi}=v, \quad v_{\xi}=-D^{-1}(c v+F(u ; \mu)) .
$$

For $c=c_{*}$, this equation possesses the equilibria $\left(U_{ \pm}, 0\right)$ and the heteroclinic orbit $\left(Q(\xi), Q_{\xi}(\xi)\right)$, which converges to $(U \pm, 0)$ as $\xi \rightarrow \pm \infty$. The equilibria $\left(U_{ \pm}, 0\right)$ are hyperbolic within $Y_{0}$, since any purely imaginary eigenvalue $\mathrm{i} k_{0}$ would result in a solution of $\operatorname{det}\left(-D k^{2}+\partial_{U} F\left(U_{0} ; 0\right)\right)=0$ with $U_{0}=U_{+}$or $U_{-}$in contradiction to Hypotheses 3 or 4 , respectively. We denote by $W_{0}^{\mathrm{u}}\left(U_{-}\right)$the unstable manifold of (4) associated with $\left(U_{-}, 0\right)$ and by $W_{0}^{\mathrm{s}}\left(U_{+}\right)$the stable manifold of (4) associated with $\left(U_{+}, 0\right)$. We claim that $W_{0}^{\mathrm{u}}\left(U_{-}\right)$and $W_{0}^{\mathrm{s}}\left(U_{+}\right)$cross transversely upon varying $c$ near $c=c_{*}$; see figure 8. More precisely, the parameter-dependent stable and unstable manifolds intersect transversely along the heteroclinic orbit in the extended phase space $(c,(u, v)) \in \mathbb{R} \times Y_{0}$ : 


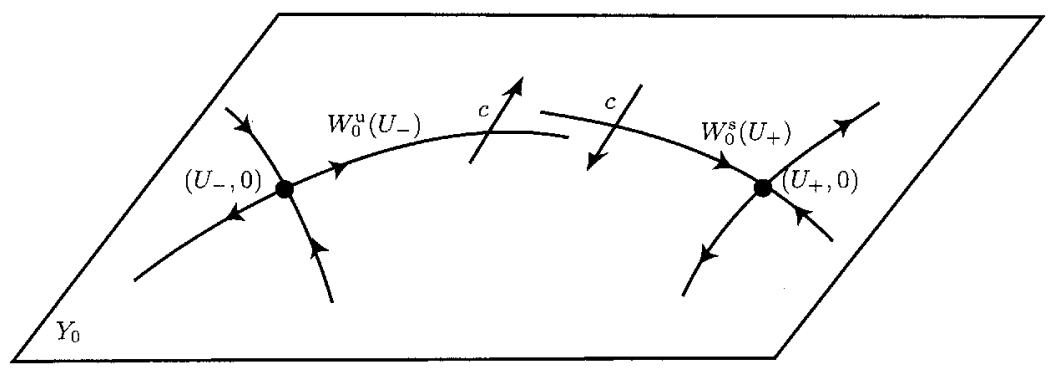

Figure 8. The transverse crossing of $W_{0}^{\mathrm{u}}\left(U_{-}\right)$and $W_{0}^{\mathrm{s}}\left(U_{+}\right)$in $Y_{0}$.

$$
\left(\bigcup_{c \sim c_{*}} W_{0}^{\mathrm{u}}\left(U_{-}\right)\right) \overline{\mathrm{a}}\left(\bigcup_{c \sim c_{*}} W_{0}^{\mathrm{s}}\left(U_{+}\right)\right)=\left\{\left(Q(\xi), Q_{\xi}(\xi)\right) ; \xi \in \mathbb{R}\right\}
$$

which we also write as $W_{0}^{\mathrm{u}}\left(U_{-}\right) \overline{\mathrm{a}}_{c} W_{0}^{\mathrm{s}}\left(U_{+}\right)$. Indeed, the dimensions of $W_{0}^{\mathrm{u}}\left(U_{-}\right)$and $W_{0}^{\mathrm{s}}\left(U_{+}\right)$add up to $2 N$ since, by Hypothesis $2, \lambda=0$ is an isolated eigenvalue and the Fredholm index of the linearization is zero; see section 2. If the two manifolds were intersecting non-transversely, the eigenvalue zero would have algebraic multiplicity larger or equal to two; see Sandstede (1998, Lemma 5.5) for more details.

Next, we address the dynamical behaviour near the rest state that undergoes the Turing instability. In the co-moving frame, the Turing bifurcation is oscillatory, and the bifurcating stationary Turing patterns become both time and spatially periodic. Fixing the temporal period $p$ and the wave speed $c$ selects a unique Turing pattern with wavenumber $k=2 \pi /(c p)$. Interpreted in terms of the spatial dynamical system (3), the Turing bifurcation is nothing other than a generic Hopf bifurcation with $S^{1}$ symmetry; recall that the symmetry is induced by the time-shift. We emphasize that the Turing bifurcation involves time-periodic functions with non-zero minimal period, and can therefore not be seen in the subspace $Y_{0}$. The Hopf frequency is given by the spatial wavenumber $k_{*}$. The complete bifurcation can be reduced to a two-dimensional centre manifold for the $\xi$-dynamics, and equation (3) restricted to the centre manifold is automatically in Hopf normal-form due to the non-trivial action of the $S^{1}$-symmetry on the critical eigenfunctions. The sign of the cubic coefficient is negative. The real part of the spatial eigenvalue crosses the imaginary axis from right to left as $\mu$ crosses through zero from left to right. The relevant computations of these signs are given in Sandstede and Scheel (1999a, 2000). A heuristic argument goes as follows. Turing patterns exist for $\mu$ positive. Within the centre manifold, there exists a small heteroclinic orbit that connects the Turing patterns with the trivial rest state $\left(U_{+}\right.$if the Turing instability occurs ahead of the front and $U_{-}$if it occurs behind the front). This small heteroclinic orbit represents a modulated front that describes Turing patterns which invade the unstable rest state. With the convention $c_{*}>0$, the stable Turing patterns have to be located at $\xi=-\infty$, and they invade the unstable rest state at $\xi=+\infty$. The heteroclinic orbit therefore converges to the Turing patterns as $\xi \rightarrow-\infty$ so that the Turing patterns have to be unstable, with respect to the $\xi$-dynamics, within the centre manifold. Hence, the cubic coefficient has to be negative. Since the Turing patterns exist for $\mu$ positive, the real part of the critical eigenvalue has to cross from right to 
left for increasing $\mu$. As before, we denote the Turing patterns by $T_{\mu, k}(\xi)$, where $k$ depends on the wave speed $c$ via $c k=2 \pi / p$.

We are now in a position to explain the existence and non-existence results stated in Theorems 3 and 4 . We begin by considering Turing instabilities ahead of the front.

\subsection{Turing instabilities ahead of the front}

Summarizing the discussion above, the primary front $Q$ corresponds to a heteroclinic orbit $\left(Q, Q_{\xi}\right)$ that connects the rest state $\left(U_{-}, 0\right)$ to the rest state $\left(U_{+}, 0\right)$ that undergoes a Hopf bifurcation when considered for the spatial dynamical system. We seek heteroclinic orbits of the spatial dynamical system (3) that connect the rest state $\left(U_{-}, 0\right)$ to the Turing pattern $T_{\mu, k}(\xi)$ that bifurcates at the Hopf bifurcation point. In other words, we seek intersections of the unstable manifold $W^{\mathrm{u}}\left(U_{-}\right)$of $\left(U_{-}, 0\right)$ and the strong stable manifold $W^{\mathrm{ss}}\left(T_{\mu, k}\right)$ of the Turing pattern. Global existence and smooth dependence on parameters of these invariant manifolds for equation (3) in a neighbourhood of the primary heteroclinic orbit $\left(Q, Q_{\xi}\right)$ have been established by Sandstede and Scheel (1999a) using exponential dichotomies. Though local existence near the rest state and the Turing pattern can be established by the variation-ofconstants formula as for ODEs, global existence is a non-trivial problem: since the initial-value problem for equation (3) is ill-posed, there is no flow or semiflow available to transport the local invariant manifolds forward or backward in $\xi$.

We illustrate the resulting bifurcation scenario in figure 9 where we plotted certain particular trajectories as well as the invariant manifolds for the spatial dynamics (3). We identified $S^{1}$-group orbits, i.e. time-shifted solutions, so that we actually consider solutions in the quotient space $Y / S^{1}$. In $Y / S^{1}$, the Hopf instability reduces to a pitchfork-type bifurcation.

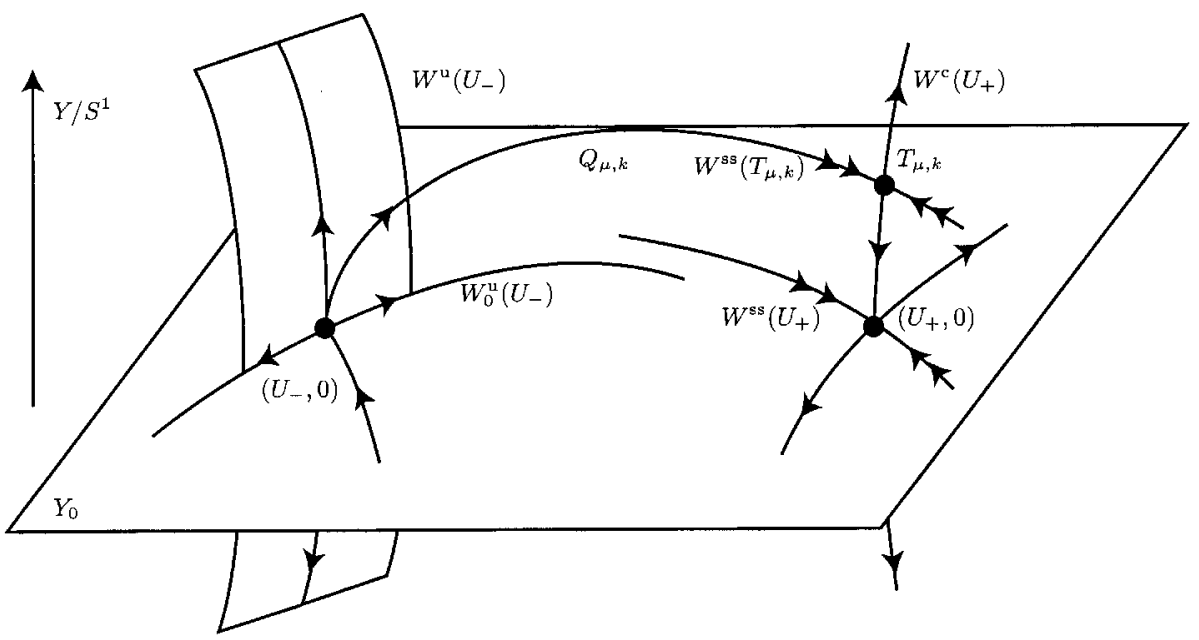

Figure 9. Turing instabilities ahead of the front. The heteroclinic bifurcation in $Y / S^{1}$ that corresponds to a Turing instability ahead of the primary front. The modulated front $Q_{\mu, k}$ arises as the intersection of the unstable manifold of $U_{-}$ and the strong stable manifold of the Turing pattern $T_{\mu, k}$. 
First, we claim that the extended unstable manifold of $U_{-}$intersects the extended strong stable manifold of $U_{+}$transversely in the full space $Y$ at $\mu=0: W^{\mathrm{u}}\left(U_{-}\right) \overline{\mathrm{a}}_{c}$ $W^{\text {ss }}\left(U_{+}\right)$. Note that we consider the strong stable manifold of $U_{+}$so that the centre direction along which the Turing pattern bifurcates is not taken into account. The above claim of transversality follows once again from the spectral Hypothesis 6 . Otherwise, we would obtain a solution in $Y$ to the linearized equation

$$
u_{\xi}=v, \quad v_{\xi}=D^{-1}\left(u_{t}-c v-\partial_{U} F(Q(\xi) ; 0) u\right)
$$

that decays exponentially to zero in $Y$ as $\xi \rightarrow \pm \infty$. Any such solution corresponds to an exponentially decaying eigenfunction of $L_{Q}$ associated with a purely imaginary eigenvalue. This contradicts Hypothesis 6 which precludes the existence of such eigenmodes.

Next, consider the strong stable manifold $W^{\mathrm{ss}}\left(T_{\mu, k}\right)$ of an arbitrarily chosen point on $T_{\mu, k}$. Since $T_{\mu, k}$ is close to $\left(U_{+}, 0\right)$, their strong stable manifolds are close to each other by continuous dependence. Hence, since $W^{\mathrm{u}}\left(U_{-}\right)$and $W^{\mathrm{ss}}\left(U_{+}\right)$intersect transversally as $c$ varies near $c_{*}$, so do $W^{\mathrm{u}}\left(U_{-}\right)$and $W^{\mathrm{ss}}\left(T_{\mu, k}\right)$. Thus, by transversality, there is a locally unique intersection curve of these two manifolds for every $\mu>0$ sufficiently small and some wave speed $c$ close to $c_{*}$. This proves the existence part of theorem 3. The details of the aforementioned arguments are similar to those given in Sandstede and Scheel (1999a) for the case of homoclinic bifurcations.

We discuss the spectral stability of the bifurcating modulated fronts in section 6 .

We remark that, for each given minimal temporal period chosen as above, there is also a continuum of modulated waves that connect $U_{-}$with $U_{+}$. These additional waves are different from the persisting primary travelling wave. They arise as intersections of the unstable manifold $W^{\mathrm{u}}\left(U_{-}\right)$with the stable manifold $W^{\mathrm{s}}\left(U_{+}\right)$; see figure 9. Note that $W^{\mathrm{s}}\left(U_{+}\right)$also contains the weak stable direction that coincides with the centre direction at the bifurcation point. Thus, there is a continuum of different intersections between $W^{\mathrm{u}}\left(U_{-}\right)$and $W^{\mathrm{s}}\left(U_{+}\right)$that can be parameterized by their amplitude, taken in the centre direction, measured in a certain fixed crosssection that is transverse in $Y$ to the primary front.

\subsection{Turing instabilities behind the front}

Finally, we demonstrate the non-existence of modulated fronts if the Turing instability occurs behind the front. The geometry of the relevant heteroclinic bifurcation is illustrated in figure 10 . Hypothesis 6 implies that $W^{\mathrm{cu}}\left(U_{-}\right) \overline{\mathrm{a}}_{c} W^{\mathrm{s}}\left(U_{+}\right)$in $Y$ where $W^{\text {cu }}\left(U_{-}\right)$denotes the centre-unstable manifold of $U_{-}$that consists of all solutions with weak exponential growth as $\xi \rightarrow-\infty$. Upon varying $\mu$, the unique intersection persists by transversality. One such intersection, however, is given by the heteroclinic orbit between $U_{-}$and $U_{+}$that persists within $Y_{0}$. By uniqueness, there are then no other intersections. On the other hand, any modulated wave connecting $T_{\mu, k}$ to $\left(U_{+}, 0\right)$ would have to lie in the aforementioned intersection. This proves Theorem 4.

\section{Spectra of modulated waves}

In this section, we investigate the stability properties of the modulated fronts that invade a stable Turing pattern. We consider the linearization

$$
V_{t}=D V_{\xi \xi}+c V_{\xi}+\partial_{U} F\left(Q_{\mu, k}(t, \xi) ; \mu\right) V,
$$




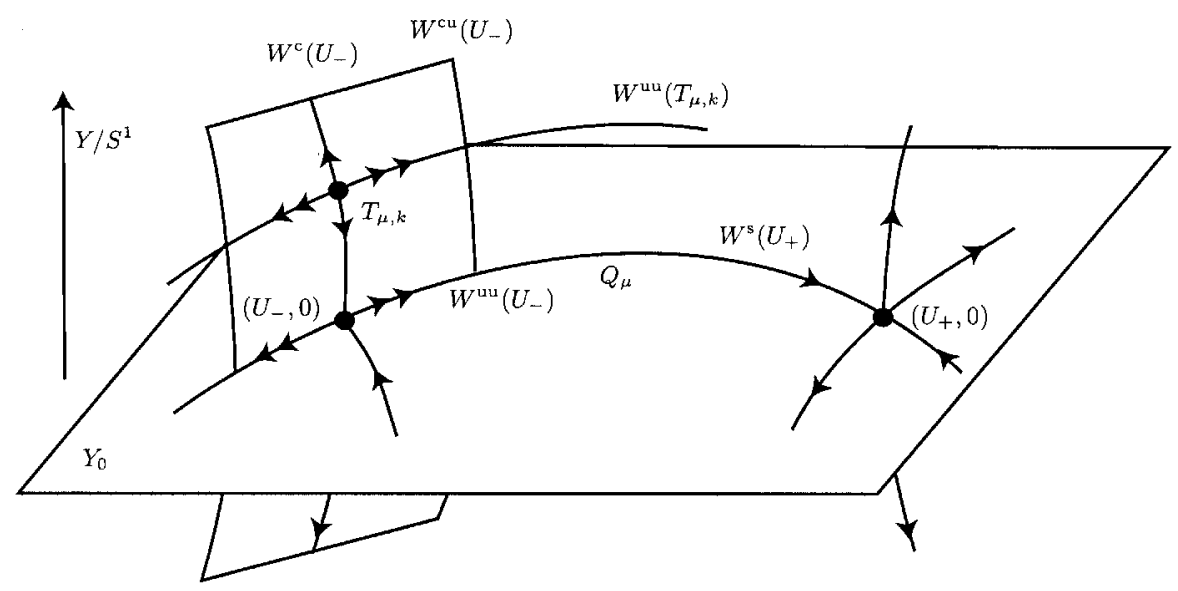

Figure 10. Turing instabilities behind the front. The heteroclinic bifurcation in $Y / S^{1}$ that corresponds to a Turing instability behind the primary front. The unstable manifold of the Turing pattern cannot intersect the stable manifold of the rest state $U_{+}$.

which is a linear parabolic equation with time-periodic coefficients. We denote the associated temporal period map by $\Phi: \mathcal{L}^{2}\left(\mathbb{R}, \mathbb{R}^{N}\right) \rightarrow \mathcal{L}^{2}\left(\mathbb{R}, \mathbb{R}^{N}\right)$, omitting the dependence on $\mu, k$. We shall prove that $\{\lambda ;|\lambda| \geq 1, \lambda \neq 1\}$ belongs to the resolvent set of the bounded operator $\Phi$. With $\alpha=p \log \lambda$, we define $\tilde{V}=\mathrm{e}^{-\alpha t} V$. Upon substituting this expression into equation (5), we get

$$
\tilde{V}_{t}=D \tilde{V}_{\xi \xi}+c \tilde{V}+\partial_{U} F\left(Q_{\mu, k}(t, \xi) ; \mu\right) \tilde{V}+\alpha \tilde{V}
$$

The key is to again write the parabolic equation as an ill-posed dynamical system in the spatial variable $\xi$ :

$$
\tilde{v}_{\xi}=\tilde{w}, \quad \tilde{w}_{\xi}=D^{-1}\left(\tilde{v} t-c \tilde{w}-\partial_{U} F\left(Q_{\mu, k}(t, \xi) ; \mu\right) \tilde{v}-\alpha \tilde{v}\right)
$$

on $Y$. In Sandstede and Scheel (1999c, Theorem 3), we proved Palmer's theorem for modulated waves. We demonstrated that invertibility of $\Phi-\lambda$ is equivalent to the existence of exponential dichotomies for equation (6) on $\mathbb{R}$; see Peterhof et al. (1997) for a definition of exponential dichotomies in the case of ill-posed problems. Roughly speaking, an exponential dichotomy is a decomposition of the phase space $Y$ into stable subspaces $E^{\mathrm{s}}(\xi)$ and unstable subspaces $E^{\mathrm{u}}(\xi)$ such that, for any initial value in $E^{\mathrm{s}}\left(\xi_{0}\right)$, there exists a solution on $\left[\xi_{0},+\infty\right)$ that decays exponentially as $\xi \rightarrow \infty$. Similarly, for any initial condition in $E^{\mathrm{u}}\left(\xi_{0}\right)$, there exists a solution on $\left(-\infty, \xi_{0}\right]$ that decays exponentially as $\xi \rightarrow-\infty$. We also consider exponential dichotomies on $\mathbb{R}_{+}$and $\mathbb{R}_{-}$where the above decomposition and the solutions are only required to exist for $\xi \in \mathbb{R}_{+}$and $\xi \in \mathbb{R}_{-}$, respectively. Dichotomies on $\mathbb{R}_{+}$and $\mathbb{R}_{-}$imply the existence of a dichotomy on the entire real line $\mathbb{R}$ if, and only if, they intersect transversely at $\xi=0$ so that $E_{+}^{\mathrm{s}}(\xi=0) \oplus E_{-}^{\mathrm{u}}(\xi=0)=Y$.

We begin by investigating the asymptotic equations that account for the essential spectrum of $\Phi$. For $\xi \rightarrow-\infty$, we obtain

$$
\tilde{v}_{\xi}=\tilde{w}, \quad \tilde{w}_{\xi}=D^{-1}\left(\tilde{v}_{t}-c \tilde{w}-\partial_{U} F\left(U_{-} ; \mu\right) \tilde{v}-\alpha \tilde{v}\right) .
$$


Since we assumed that $U_{-}$is a stable rest state for the parabolic equation, the above equation has an exponential dichotomy for all $\alpha$ with $\operatorname{Re} \alpha \geq-\delta$ for some small positive $\delta$. Note that the stable and unstable subspaces are independent of $\xi$ since the equation does not depend upon $\xi$. The dichotomy for the asymptotic equation persists as a dichotomy on $\mathbb{R}^{-}$for the full equation (6) by the roughness theorem for exponential dichotomies proved by Peterhof et al. (1997). We denote the associated stable and unstable subspaces by $E_{-}^{\mathrm{s}}(\xi ; \mu, \alpha)$ and $E_{-}^{\mathrm{u}}(\xi ; \mu, \alpha)$, respectively. The exponential decay rates of the associated solutions are uniformly bounded away from zero for $\alpha$ with $\operatorname{Re} \alpha>-\delta$. In particular, any bounded solution to equation (6) lies in $E^{\mathrm{u}}\left(\xi_{0}\right)$ at spatial time $\xi=\xi_{0}$.

The situation at $\xi=+\infty$ is slightly more complicated since hyperbolicity fails at the moment of bifurcation. We then cite the results in Sandstede and Scheel (2000, sections 5.1 and 5.2) where a similar problem has been considered in the context of spectral stability of a modulated pulse that is asymptotic to a Turing pattern. We outline the arguments, and refer to Sandstede and Scheel (2000) for a more thorough discussion. In a steady frame, centre-manifold reductions and normal-form arguments for the spatial $x$-dynamics on the space of time-independent functions reduce the reaction-diffusion problem to a complex Ginzburg-Landau equation with real coefficients in an appropriate scaling. For the Ginzburg-Landau equation, the spectrum can be computed explicitly. We write $T_{\mu, k}(x)$ for the Turing patterns and study the linearization $L_{T_{\mu, k}}-\lambda$ of the reaction-diffusion system linearized about $T_{\mu, k}$. Associated with $L_{T_{\mu, k}}-\lambda$ is the linearized equation

$$
u_{x}=v, \quad v_{x}=D^{-1}\left(-\partial_{U} F\left(T_{\mu, k}(x) ; \mu\right) u+\lambda u\right) .
$$

A complex number $\lambda$ is in the spectrum of $L_{T_{\mu, k}}$ if, and only if, equation (7) has a purely imaginary Floquet exponent. There exist two curves, $\lambda_{\mathrm{cr}}(\rho ; \mu, k)$ and $\lambda_{\mathrm{st}}(\rho ; \mu, k)$, such that, in a neighbourhood of $\rho=0$ and $\lambda=0$, we have

(i) $\lambda_{\mathrm{cr}}(\rho ; \mu, k)>\lambda_{\mathrm{st}}(\rho ; \mu, u)$ for $\mu>0$;

(ii) $\lambda_{\mathrm{cr}}\left(\rho ; 0, k_{*}\right)=\lambda_{\mathrm{st}}\left(\rho ; 0 . k_{*}\right)$ is the dispersion relation at the bifurcation point, parameterized over the spatial wavenumber $\rho+k_{*}$;

(iii) $\lambda_{\mathrm{cr}}^{\prime}(0 ; \mu, k)=0$, and $\lambda_{\mathrm{cr}}^{\prime \prime}(0 ; \mu, k)$ is positive for $\mu<\mu_{\mathrm{st}}(k)$ and negative for $\mu>\mu_{\mathrm{st}}(k)$;

(iv) $\gamma=\mathrm{i} \rho$ is a purely imaginary Floquet exponent of equation (7) if, and only if, $\lambda=\lambda_{\mathrm{cr}}(\rho ; \mu, k)$ or $\lambda=\lambda_{\mathrm{st}}(\rho ; \mu, k)$.

Transforming into the moving coordinate frame, spatial oscillations measured by the imaginary part of the spatial Floquet exponent $\gamma$ translate into temporal oscillations measured by the imaginary part of the temporal Floquet exponent $\alpha$. Using these ideas, Sandstede and Scheel (2000) deduced from the above information about the spatial Floquet exponents that there are two critical curves, $\alpha_{\mathrm{cr}}(\rho ; \mu, k)$ and $\alpha_{\mathrm{st}}(\rho ; \mu, k)$, so that the ill-posed equation (7) has purely imaginary Floquet exponents precisely for $\alpha$ on one of the aforementioned two curves. Furthermore, the curves are explicitly given in terms of the dispersion relations in the steady frame:

$$
\begin{aligned}
& \alpha_{\mathrm{cr}}(\rho ; \mu, k)=\frac{2 \pi}{c k} \lambda_{\mathrm{cr}}(\rho ; \mu, k)+\mathrm{i} \rho \\
& \alpha_{\mathrm{st}}(\rho ; \mu, k)=\frac{2 \pi}{c k} \lambda_{\mathrm{st}}(\rho ; \mu, k)+\mathrm{i} \rho .
\end{aligned}
$$




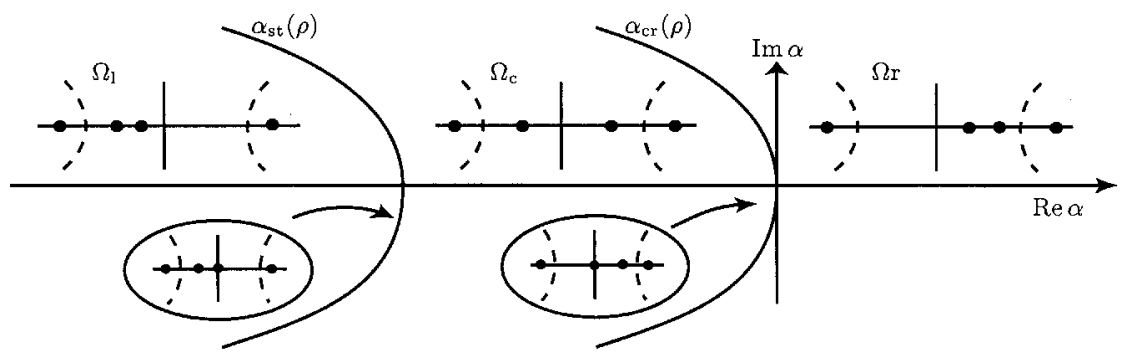

Figure 11. Upon varying $\alpha \in \mathbb{C}$ near zero, two critical spatial Floquet exponents cross the imaginary axis. Plotted are the real parts of the critical exponents and the two curves $\alpha_{\mathrm{cr}}(\rho)$ and $\alpha_{\mathrm{st}}(\rho)$.

In particular, the two curves $\alpha_{\mathrm{st}}$ and $\alpha_{\mathrm{cr}}$ divide a neighbourhood of the origin in the complex $\alpha$-plane into three connected components $\Omega_{1}, \Omega_{\mathrm{c}}$ and $\Omega_{\mathrm{r}}$. The critical spatial Floquet exponents move from left to right as $\operatorname{Re} \alpha$ increases. The first Floquet exponent crosses the axis at $\alpha=\alpha_{\text {st }}$, the second at $\alpha=\alpha_{\mathrm{cr}}$; see figure 11. It is then a consequence of Sandstede and Scheel (1999c, Proposition 1) that the essential spectrum of the modulated fronts is contained in the closed left half-plane if, and only if, the spectrum of the asymptotic Turing pattern is contained in the closed left half-plane. More precisely, the essential spectrum is bounded by the curve $\lambda(\rho)=\exp \left(\alpha_{\mathrm{cr}}(\rho) T\right)$. On this curve, the Fredholm index of $\Phi-\lambda$ changes from zero in $\Omega_{\mathrm{r}}$ to one in $\Omega_{\mathrm{c}}$ since the dimension of the stable subspace increases by one so that, in the formula $i=i_{-}-i_{+}$for the Fredholm index, the relative unstable dimension $i_{+}$decreases by one.

Since the essential spectrum is therefore stable, it suffices to exclude unstable point spectrum. For $\operatorname{Re} \alpha>0$, bounded solution to the asymptotic equation lie in the strong stable subspace $E^{\mathrm{ss}}(\xi)$, where we exclude decay with the rate of the two critical spatial Floquet exponents. We can continue this subspace in a neighbourhood of $\alpha=0$ and construct the $\alpha$ and $\xi$-dependent strong stable subspace $E_{+}^{\mathrm{ss}}(\xi ; \mu, \alpha)$ of the full linearization about the travelling wave. We investigate the intersections of $E_{+}^{\mathrm{ss}}(\xi ; \mu, \alpha)$ and $E_{-}^{\mathrm{u}}(\xi ; \mu, \alpha)$, and demonstrate that there are no such intersections for $\operatorname{Re} \alpha>0$. At $\mu=0$, there is a unique intersection at $\alpha=0$ where the two subspaces cross with non-vanishing speed in $\alpha$. This is again a consequence of Hypothesis 6. The unique intersection corresponds to the derivative of the primary front. For $\mu>0$, this unique intersection persists in a neighbourhood of $\alpha=0$. In fact, it is given by the time-derivative of the modulated wave with respect to $x=\xi+c t$ which is a solution to equation (6) for $\alpha=0$ that decays exponentially as $\xi \rightarrow+\infty$ with a rate that is uniform in $\mu$. Thus, the unique intersection is located in $\operatorname{Re} \alpha \leq 0$, and there cannot be any additional unstable eigenvalues near $\alpha=0$. This proves spectral stability of the modulated fronts. As mentioned above, more details for modulated pulses can be found in Sandstede and Scheel (2000, Section 5.3).

\section{A model problem}

We investigate a three-component reaction-diffusion system for which the assumptions of our theorems can be verified explicitly. Consider 


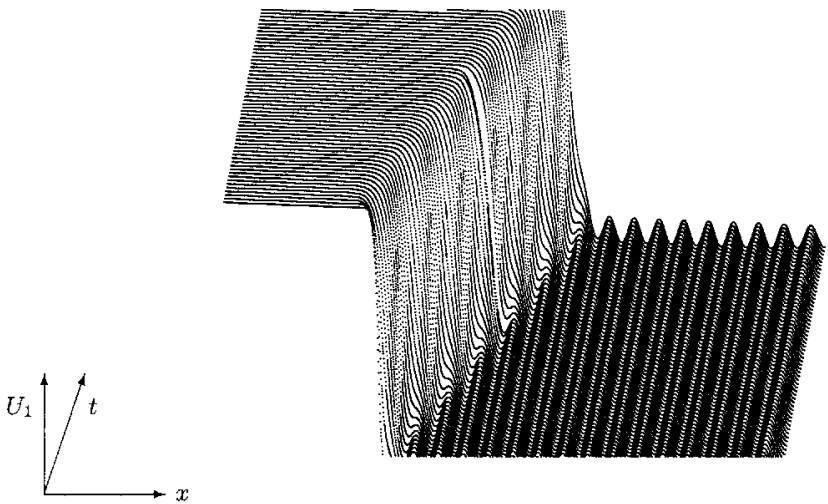

Figure 12. (Turing instabilities ahead of the front) A space-time plot of the modulated front to equation (8) that invades a Turing pattern. The parameters are $a=0.2, \varepsilon=0.2$ and $\mu=0.002$.

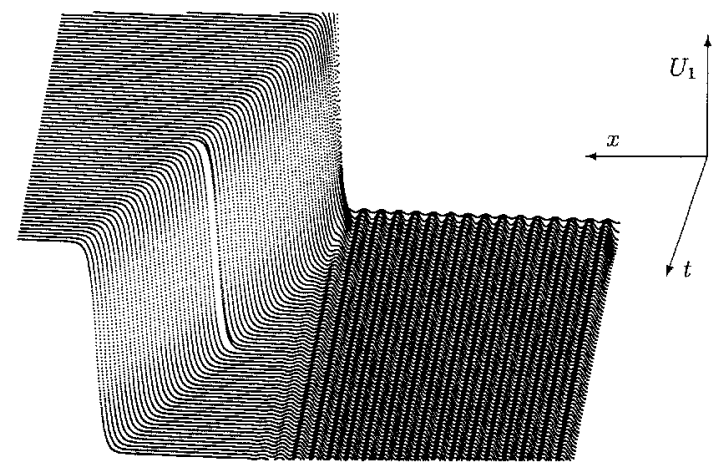

Figure 13. (Turing instabilities behind the front) A space-time plot of the front to equation (8) that leaves a Turing pattern behind. The parameters are $a=0.8$, $\varepsilon=0.2$ and $\mu=0.002$. The resulting pattern is not time-periodic in any comoving frame.

$$
\begin{aligned}
& \partial_{t} U_{1}=\partial_{x x} U_{1}+f\left(U_{1}\right)+U_{2}, \\
& \partial_{t} U_{2}=\partial_{x x} U_{2}-U_{2}+2 U_{3} \\
& \partial_{t} U_{3}=\frac{1}{6} \partial_{x x} U_{3}-\frac{1}{3} U_{2}+\frac{1}{2} U_{3}+\mu U_{3}-\left(U_{3}\right)^{3}-\varepsilon U_{1} U_{3},
\end{aligned}
$$

where $f(u)=u(1-u)(u-a)$. In the invariant subspace $U_{2}=U_{3}=0$, we recover the classical bistable scalar reaction-diffusion equation with cubic kinetics that we discussed in section 1. For $0<a<1 / 2$, it exhibits a front solution $Q_{1}(\xi)$ that connects $U_{1}=1$ at $\xi=-\infty$ to $U_{1}=0$ at $\xi=+\infty$ where $\xi=x-c_{*} t$ and $c_{*}>0$. For $1 / 2<a<1$, the front connects $U_{1}=0$ to $U_{1}=1$ again with positive wave speed. The equilibrium $U=(1,0,0)$ is exponentially stable at $\mu=0$ for any fixed positive $\varepsilon>0$. The equilibrium $U=(0,0,0)$ undergoes a non-degenerate supercritical Turing bifurcation upon varying $\mu$ close to zero. It is straightforward to verify that 
Hypotheses 2, 3 and 5 are satisfied for $0<a<1 / 2$, while Hypotheses 2, 4 and 5 are met for $1 / 2<a<1$.

It remains to prove that Hypothesis 6 is satisfied. The linearization about $U=\left(Q_{1}(\xi), 0,0\right)$ in a moving frame reads:

$$
\begin{aligned}
& \partial_{t} V_{1}=\partial_{\xi \xi} V_{1}+c \partial_{\xi} V_{1}+\partial_{U_{1}} f\left(Q_{1}(\xi)\right) V_{1}+V_{2}, \\
& \partial_{t} V_{2}=\partial_{\xi \xi} V_{2}+c \partial_{\xi} V_{2}-V_{2}+2 V_{3}, \\
& \partial_{t} V_{3}=\frac{1}{6} \partial_{\xi \xi} V_{3}+c \partial_{\xi} V_{3}-\frac{1}{3} V_{2}+\frac{1}{2} V_{3}+\mu V_{3}-\varepsilon Q_{1}(\xi) V_{3} .
\end{aligned}
$$

With a small exponential weight $V=\mathrm{e}^{\eta \xi} W$, we obtain the eigenvalue problem

$$
\begin{aligned}
\lambda W_{1}= & \partial_{\xi \xi} W_{1}+(c-2 \eta) \partial_{\xi} W_{1}+\left(\eta^{2}-c \eta\right) W_{1}+\partial_{U_{1}} f\left(Q_{1}(\xi)\right) W_{1}+W_{2}, \\
\lambda W_{2}= & \partial_{\xi \xi} W_{2}+(c-2 \eta) \partial_{\xi} W_{2}+\left(\eta^{2}-c \eta\right) W_{2}-W_{2}+2 W_{3}, \\
\lambda W_{3}= & \frac{1}{6} \partial_{\xi \xi} W_{3}+\left(c-\frac{\eta}{3}\right) \partial_{\xi} W_{3}+\left(\frac{\eta^{2}}{6}-c \eta\right) W_{3}-\frac{1}{3} W_{2} \\
& +\frac{1}{2} W_{3}+\mu W_{3}-\varepsilon Q_{1}(\xi) W_{3} .
\end{aligned}
$$

This equation is of skew-product form. Upon setting $W_{2}=0$, the first equation has precisely one eigenvalue in $\operatorname{Re} \lambda \geq 0$, namely $\lambda=0$, which is algebrically simple, with the eigenmode $\partial_{\xi} Q_{1}(\xi)$. For all $\eta>0$ small, the linear system (9) is hyperbolic for $\operatorname{Re} \lambda>0$ at $\xi= \pm \infty$, eigenvalues are zeros of the Evans function $E(\lambda ; \varepsilon, \eta)$. For $\varepsilon=0$, the $\left(W_{2}, W_{3}\right)$-components of equation (9) are autonomous. Using the results by Gardner and Zumbrun (1998) and Kapitula and Sandstede (1998), it is not hard to see that $E$ can be extended smoothly up to and including $\varepsilon=0$ and $\eta=0$. For $(\varepsilon, \eta)=0$, however, the Evans function is non-zero since the equation is autonomous. By continuity, the Evans function remains non-zero for all small positive values of $(\varepsilon, \eta)$. This proves the absence of critical or unstable point spectrum according to Hypothesis 6 . We conclude this section with some numerical snapshots of the dynamics for small $\mu, \varepsilon>0$.

\section{The Gray-Scott model}

The Gray-Scott model

$$
\begin{aligned}
& \partial_{t} U_{1}=d_{1} \partial_{x x} U_{1}-U_{1} U_{2}^{2}+F\left(1-U_{1}\right) \\
& \partial_{t} U_{2}=d_{2} \partial_{x x} U_{2}+U_{1} U_{2}^{2}-(F+k) U_{1}
\end{aligned}
$$

is a model for an autocatalytic chemical reaction; it supports oscillations in the kinetics. A rich variety of dynamical phenomena have been discovered in this reaction-diffusion model; we refer to the comprehensive list of references in Mazin et al. (1996). In particular, localized stable pulses have been observed that can undergo various kinds of bifurcations including a self-replicating mechanism which has recently received much attention. In the region of bistability, there exist fronts that travel to either one of the two stable patterns given by 


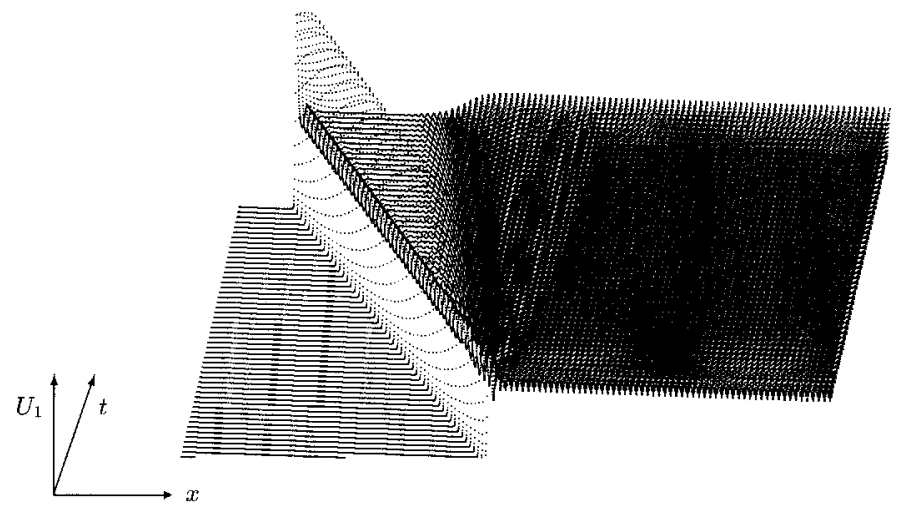

Figure 14. We solved the Gray-Scott model on the finite interval $[0,30]$ with Neumann boundary conditions. The parameter values are $k=0.04$ and $F=0.021$. The figure shows a space-time plot of the $U_{1}$-component of the front that leaves a Turing pattern behind.

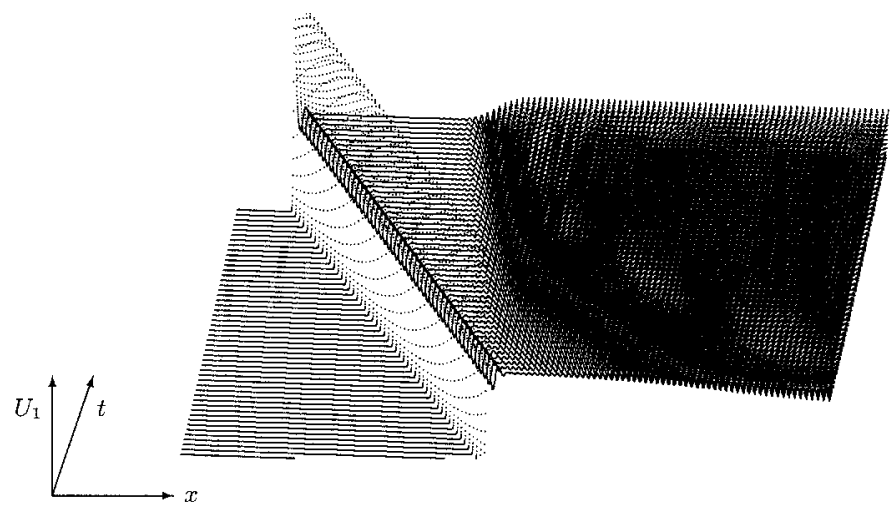

Figure 15. We solved the Gray-Scott model for the same parameter values as in figure 14. Initially, the small Turing front has a larger velocity than the primary front. As the Turing pattern approaches the primary front, however, the Turing pattern is pushed back by the front and eventually left behind.

$$
U_{-}=\left(\frac{1}{2}(1-\sqrt{\Delta}), \frac{F}{2(F+k)}(1+\sqrt{\Delta})\right), \quad U_{+}=(1,0)
$$

where $\Delta=1-4(F+k)^{2} / F$. The rest state $U_{-}$can undergo a Turing instability that is either subcritical or supercritical depending on the parameters. We produce numerical evidence for a line in $(F, k)$-parameter space, where the system undergoes an instability of the type considered in Theorem 4.

To illustrate these numerical findings, we fix the parameters

$$
d_{1}=1.0 \times 10^{-5}, \quad d_{2}=6.0 \times 10^{-5} .
$$

We have then numerically computed a curve in the $(F, k)$-parameter space where the system undergoes a supercritical Turing instability. We have also numerically com- 
puted the front which appears to be stable as long as the parameters do not cross the aforementioned Turing-instability curve. It appears as if the Turing instability is supercritical only in the regime where the front moves away from the destabilizing rest state; this precludes the existence of the stable modulated fronts since only Theorem 4 applies. We present space-time plots of direct numerical simulations that confirm the predicted blocking of the Turing patterns by the front. In particular, it appears as if modulated fronts do not exist as predicted by Theorem 4 .

In the case of a subcritical Turing instability, a standing interface between standing large-amplitude Turing patterns and the homogeneous zero rest state is formed from small perturbations of the primary unstable front. We suspect that this large interface is connected, in the parameter space, to an unstable modulated front that is close to the primary front and leaves unstable Turing patterns behind; see the discussion of subcritical bifurcations in section 9 .

\section{Discussion}

In this section, we discuss various extensions of the results presented above as well as some open problems.

\subsection{Subcritical Turing instabilities ahead or behind a front}

We begin by discussing subcritical Turing bifurcations. A subcritical Turing instability ahead of the front creates a continuum of modulated fronts that invade an unstable Turing pattern of fixed wavelength; see figure 16. Since the Turing pattern is unstable, so are the modulated fronts. If, on the other hand, the subcritical Turing instability occurs behind the front, then modulated fronts cannot exist; see figure 17.

An interesting case is the degenerate Turing instability where the cubic coefficient vanishes. An unfolding of the degeneracy requires two parameters. It is then possible that a stable rest state and a stable Turing pattern coexist; in the phase space $Y$, they

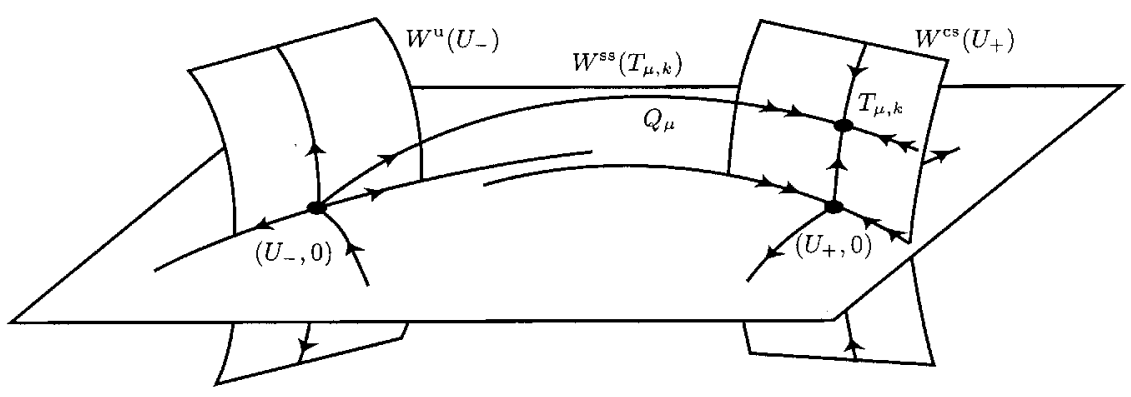

Figure 16. Subcritical Turing bifurcation ahead of the front. The heteroclinic bifurcation in $Y / S^{1}$ that is associated with a subcritical Turing instability ahead of the primary front. One of the heteroclinic orbits that connect $U_{-}$with the temporally unstable Turing pattern near $U_{+}$is plotted. Note that there is a continuum of such modulated fronts; we plotted the heteroclinic orbit that lies in the strong stable manifold of the Turing pattern. 


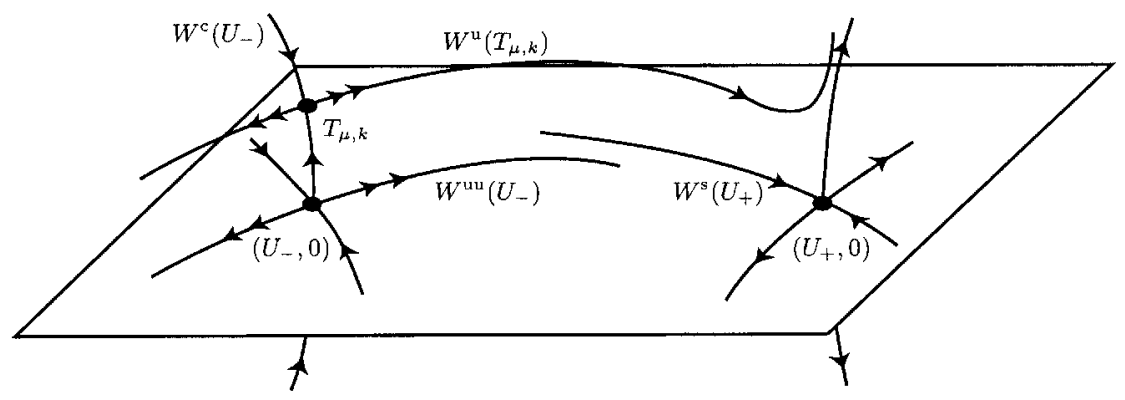

Figure 17. Subcritical Turing bifurcation behind the front. The heteroclinic bifurcation in $Y / S^{1}$ that is associated with a subcritical Turing instability behind the primary front. This demonstrates the non-existence of heteroclinic orbits that connect one of the temporally unstable Turing patterns behind the front with the rest state $U_{+}$ahead.

are separated by an unstable Turing pattern. The additional stable Turing pattern can be invaded by the front, but not left behind. The proof is the same as for the supercritical Turing instability.

\subsection{Fronts that connect stable and unstable rest states}

We may also consider fronts that connect a stable and an unstable rest state with each other. We are then interested in the situation where the stable rest state undergoes a supercritical Turing instability. There are then quite a number of different cases that have to be analysed.

First, suppose that the Turing instability occurs behind the front. The bifurcation scenario depends then crucially on the essential spectrum of the unstable rest state $U_{+}$ahead of the front-recall that we assumed that the front connects the rest state $U_{-}$that undergoes the Turing instability with a different, already unstable, rest state $U_{+}$ahead.

If the essential spectrum of the unstable state $U_{+}$does not involve the temporal frequency of the Turing instability at the state $U_{-}$, everything computed in the comoving frame of the front, then we expect to encounter the same situation that we consider in section 5; in particular, modulated fronts do not bifurcate. For rest state $U_{+}$that destabilize inside the space $Y_{0}$ of time-independent functions, this claim is illustrated in figure 18 .

Sherratt (1998) investigated a series of certain caricature problems numerically and analytically for Turing instabilities behind the front. He observed several modulated waves of different wave speed that are glued together. This is in accordance with our predictions as he implicitly assumed that the unstable rest state has no unstable eigenmodes with the same temporal frequency as the critical modes of $U_{-}$.

If, on the other hand, the unstable state $U_{+}$had already undergone a single Turing instability with the same temporal frequency as the state $U_{-}$, then a continuum of modulated fronts that connect the Turing pattern near $U_{-}$with the unstable rest state $U_{+}$bifurcate. The reason for this behaviour is that, in the relevant figure 10 , the unstable vertical direction at $U_{+}$is stable in the situation considered here. 


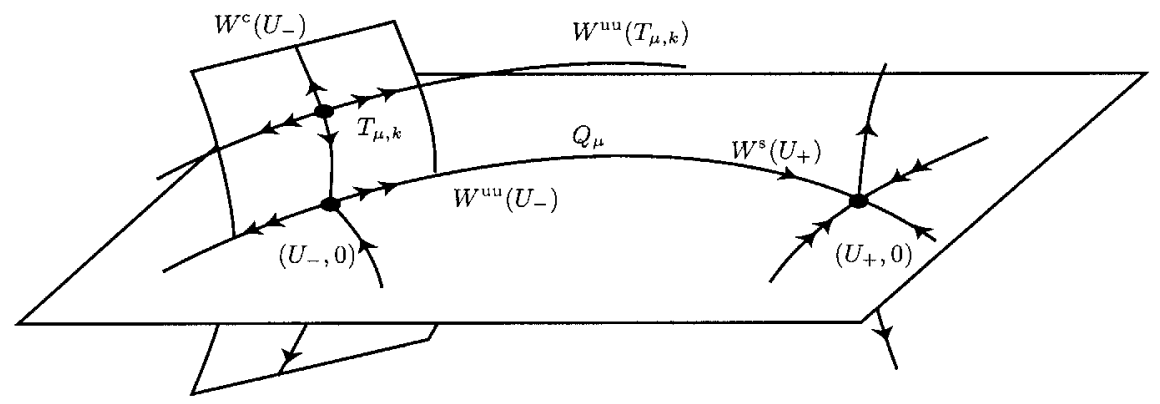

Figure 18. Turing instability behind the front. The front connects the rest state $U_{-}$ that just underwent a supercritical Turing instability, with the unstable rest state $U_{+}$that destabilized inside the subspace $Y_{0}$. Modulated fronts do not exist near such an instability.

Second, suppose that the Turing instability occurs ahead the front. The expected results are then similar to those given above, and we omit the details.

\subsection{Hopf and Turing-Hopf instabilities ahead or behind a front}

We have discussed the effects that a Turing instability of a homogeneous rest state has on a front that connects this rest state to a different stable rest state. There are two other interesting cases that lead to essential instabilities, namely Hopf and Turing-Hopf instabilities in the steady frame. The basic patterns that are created near the unstable $U_{0}$ are of the form $T_{\mu, k}(t, x)=U_{0}+A(\mu, k) \mathrm{e}^{\mathrm{i}(k x+\omega(k) t)}+$ c.c. where $\omega(k)$ denotes the linear dispersion relation and $A(\mu, k)$ is small nonlinear complex amplitude. We have $k \sim 0$ for Hopf instabilities and $k \sim k_{*} \neq 0$ for Turing-Hopf instabilities. In a moving frame, the patterns are of the form $T_{\mu, k}(\xi)=U_{0}+$ $A(\mu, k) \mathrm{e}^{\mathrm{i}(k \xi+(\omega(k)+c k) t)}$. Hence, they are time-periodic with period $p=2 \pi /(\omega(k)+c k)$ where we excluded the resonance case when $c k_{*}=\omega\left(k_{*}\right)$; these resonances are similar to the case $c=0$ for Turing instabilities. The local bifurcation near the unstable state, in a moving frame and again considered for the spatial $\xi$ dynamics on $p$-periodic functions, is a Hopf bifurcation with $S^{1}$-symmetry. Stability of the bifurcating pattern in spatial dynamics within the two-dimensional centre manifold depends on $c$ and the group velocity of the bifurcating basic patterns $c_{\mathrm{gr}}=\mathrm{d} \omega / \mathrm{d} k$. Fronts may leave basic patterns with positive relative group velocity $c_{\mathrm{gr}}-c>0$ behind and may invade basic patterns with negative relative group velocity $c_{\mathrm{gr}}-c<0$. Other modulated fronts do not exist.

The stability of the modulated fronts is again a consequence of the stability of the primary front in weighted spaces together with the stability of the basic state. Stability properties of the basic state in a moving frame are inherited from the stability properties in the steady frame. The stability criteria in the steady frame, however, are slightly different compared to Turing instabilities, and the Eckhaus boundary depends crucially on the nonlinear dispersion. 


\subsection{Vanishing wave speed $c=0$}

As mentioned above, there are two other interesting cases that we did not analyse in this paper.

First, we may consider Turing or Hopf instabilities of standing pulses that have wave speed zero. This bifurcation is of codimension-one since the reaction-diffusion system is reversible for $c=0$. We expect that stable pulses bifurcate that converge to a basic pattern as $\xi \rightarrow \pm \infty$.

Second, in the case of fronts or pulses, it is of interest to understand the case when the wave speed $c_{*}$ passes through zero (or through $\omega_{*} / k_{*}$ for Turing-Hopf instabilities). Unfolding the instability of the asymptotic state and the crossing of the wave speed through zero requires two parameters. For Turing bifurcations, we expect locking phenomena, while we believe that the speed of the bifurcating waves passes through zero transversely for Hopf instabilities. We discuss these two cases in more detail.

In the Turing case, a standing interface between a family of Turing patterns and a homogeneous state is of codimension-zero and therefore robust. A physical description of this pinning effect has been given by Pomeau (1986). Geometrically, in spatial dynamics, the heteroclinic orbit between the normally hyperbolic two-dimensional family of periodic orbits and a hyperbolic equilibrium is transverse and persists upon perturbation. For instance, upon considering the spatial dynamics of a twocomponent reaction-diffusion system, the stable homogeneous state has a twodimensional stable manifold while each Turing pattern admits one unstable Floquet exponent in addition to the two trivial neutral exponents associated with the derivative of the pattern and the derivative with respect to the spatial period. The intersection of the three-dimensional unstable manifold of the family of Turing patterns and the two-dimensional stable manifold of the homogeneous state is typically transverse. In contrast, varying the parameters (such as $a$ in the above example) would typically force an interface between homogeneous rest states to move with wave speed $c(a)$ where $c^{\prime}(a) \neq 0$. Here, however, the standing interface between rest state and Turing patterns persists with wave speed zero, while the homogeneous rest state selects phase and wave number of the coexisting Turing pattern. Using a $\lambda$ lemma type argument, Coullet et al. (2000) established that transversality of the aforementioned intersection implies the existence of steady patterns that resemble Turing patterns over a large bounded spatial interval, but are localized so that the spatially homogeneous state dominates at $\pm \infty$.

On the other hand, we expect that the interval of parameter values for which such a standing interface exists is exponentially small in the amplitude of the overall system. For example, if both, homogeneous state and Turing pattern, emerge in a local degenerate Turing instability, normal-form transformations would add an additional imperfect $S^{1}$-symmetry to the system which makes the heteroclinic orbits discussed above non-transverse. Only flat terms, beyond normal form, can render a transverse heteroclinic orbit. Only for interfaces that connect small-amplitude Turing patterns to a large-amplitude homogeneous state do we expect a locking region with width of finite order in the amplitude of the Turing pattern.

The situation for Hopf instabilities is different. Heteroclinic orbits between timeperiodic basic pattern, travelling wave trains, and homogeneous rest states are of codimension-one. Since we consider the spatial dynamics on time-periodic functions, the unstable dimension is infinite. Still, we can count relative unstable dimensions (see Sandstede and Scheel 1999c). If we set the relative unstable dimension of the 
stable homogeneous state to zero, the relative unstable dimension of the timeperiodic pattern depends again on the group velocity. For a stable wave with negative group velocity, the relative unstable dimension is -1 . If we include the phase of the periodic pattern, we end up with a centre-unstable manifold of the periodic pattern and a stable manifold of the homogeneous state so that the dimensions of their tangent spaces add up to the dimension of the entire space. Intersections are necessarily two-dimensional due to temporal and spatial shift symmetries. We have only one additional parameter, the temporal period, so that heteroclinic intersections are of codimension-one: we expect that the wave speed is generically non-zero, in contrast to the situation for Turing patterns. For a stable wave with positive group velocity, the relative unstable dimension is 0 , by reversibility. Heteroclinic intersections are of codimension-zero. Still different are standing waves which are spatially and temporally periodic and also symmetric with respect to spatial reflections. They are typically parameterized by the spatial wave number $k$. Each standing wave possesses a double zero eigenvalue corresponding to space- and time-shift symmetry. The relative unstable dimension is therefore -1 , due to reflection symmetry, and the relative centre-unstable dimension is +1 . Heteroclinic intersections with the stable manifold of the homogeneous state are at least two-dimensional due to time- and space-shift symmetry. Together with the parameter $k$, we may therefore get transverse intersections so that standing waves and homogeneous states typically coexist. Using similar arguments as in Coullet et al. (2000), we expect that localized timeperiodic standing waves may occur as well.

\subsection{Beyond modulated fronts}

As implicitly mentioned in Hypothesis 6, the Turing instability is of convective nature in a moving frame so that perturbations decay pointwise. In exponentially weighted spaces, the instability disappears.

Hence, a primary front that invades a Turing-unstable homogeneous rest state is therefore stable in an exponential weight of the form $1+\mathrm{e}^{\eta \xi}$ for any $\eta>0$ small. As in Sattinger (1976), the primary front beyond the Turing instability is also nonlinearly stable in the aforementioned exponentially weighted space. The nonlinear stability of the bifurcating modulated fronts that invade the Turing patterns is a more subtle issue. Nonlinear stability of the Turing patterns follows from the spectral information derived here using the method of diffusive stability (see Eckmann et al. 1997, Schneider 1998). Nonlinear stability of the modulated front, however, is an open problem.

More interesting is the case of a Turing instability behind the front. We demonstrated that modulated fronts do not bifurcate near such an instability. Beyond the Turing instability, the primary front is again linearly (convectively) stable in an exponentially weighted space. This time, however, the weight is exponentially decreasing. Therefore, nonlinear stability does not immediately follow from linear stability since the nonlinearity is not differentiable in the function spaces of exponentially growing functions; see, however, Pego and Weinstein (1994) for a situation where this difficulty could be resolved. We continue to discuss Turing instabilities behind the primary front and comment separately on sub- and supercritical bifurcations.

In the subcritical case, where the nonlinearity amplifies linear growth, the primary front is nonlinearly unstable, and the system typically leaves a small neighbourhood 
of the primary front. In numerical simulations of the Gray-Scott model, a standing interface between the rest state and a large-amplitude Turing pattern appears to be the typical final state.

In the supercritical case, numerical simulations of the Gray-Scott model provide evidence that the front of the Gray-Scott model is nonlinearly convectively stable beyond the onset to instability as it pushes the Turing pattern away; see figures 14 and 15. After a sufficiently long time, the dynamics behind the front appears to decouple from the primary front. Small modulated fronts of Turing patterns that invade the unstable rest state govern the dynamics; the existence of such small Turing fronts has been established by Hărăgus-Courcelle and Schneider (1999). The velocity of the small Turing fronts depends on decay properties of the initial perturbation; eventually, the small Turing fronts pick up the critical wave speed given by the linear dispersion relation.

The results presented in this article should be considered as a first step towards a more complete understanding of the dynamics near an essential instability. The approach that we adopted here captures only those solutions that have a 'nice' well-defined temporal behaviour. In particular, we were able to describe all solutions that are modulated, i.e. time-periodic in an appropriate moving frame.

\subsection{The numerical computation of essential instabilities and modulated fronts}

Finally, we comment briefly on some numerical aspects.

To locate essential instabilities, we have to compute the essential spectrum of a front. The most efficient way to compute this set is to calculate the spectra of the asymptotic homogeneous rest states. Using Fourier transform, this calculation reduces to solving an algebraic problem. We emphasize that it is in general not possible to compute the essential spectrum by truncating the real line to some large but bounded interval with Neumann or Dirichlet boundary conditions, say, and to then compute the spectrum of the linearization about the front numerically utilizing some discretization scheme: Sandstede and Scheel (1999b) proved that this procedure does not reproduce the essential spectrum of the front. Point spectrum, however, can be computed using truncation to a bounded interval-we remark that the boundary conditions can sometimes introduce additional eigenvalues that are not present for the full problem on the real line.

In some of the cases that we studied, the bifurcating modulated fronts are unstable, and can therefore not be computed using direct simulations. The approach we had adopted to show their existence, however, gives a clue as to how to compute such fronts numerically: they can be found by seeking heteroclinic orbits in the space of time-periodic functions. This requires to set up an appropriate boundary-value problem formulation in the infinite-dimensional space of time-periodic functions combined with a subsequent Galerkin approximation to reduce to a boundaryvalue problem posed on a finite-dimensional space. Afterwards, packages such as AUT097 can be used to solve the resulting boundary-value problem. We refer to Lord et al. (2000) for more details as well as convergence and stability proofs for this approach. 


\section{Acknowledgements}

B. Sandstede was partially supported by the NSF under grant DMS-9971703 and by the Ohio State University through a seed grant. A. Scheel gratefully acknowledges stimulating discussions with P. Coullet during a visit at the Institut Nonlinéaire de Nice. In particular, the authors thank P. Coullet for pointing out that interfaces between standing waves and homogeneous states should be of codimension-zero.

\section{References}

Coullet, P., Riera, C., and Tresser, C., 2000, Stable localized structures in one dimension. Physical Review Letters, 84: 3069-3072.

Eckmann, J.-P., Wayne, C. E., and Wittwer, P., 1997, Geometric stability analysis for periodic solutions of the Swift-Hohenberg equation. Communications in Mathematical Physics, 190: 173-211.

Gardner, R. A., and Zumbrun, K., 1998, The gap lemma and geometric criteria for instability of viscous shock profiles. Communications in Pure and Applied Mathematics, 51: 797-855.

Golubitsky, M., and Schaeffer, D. G., 1985, Singularities and Groups in Bifurcation Theory I (New York: Springer-Verlag).

Hale, J. K., Peletier, L. A., and Troy, W. C., 1999, Stability and instability in the Gray-Scott model: the case of equal diffusivities. Applied Mathematics Letters, 12(4): 59-65.

Hale, J. K., Peletier, L. A., and Troy, W. C., 2000, Exact homoclinic and heteroclinic solutions of the Gray-Scott model for autocatalysis. SIAM Journal of Applied Mathematics, 61: 102-130.

Hărăgus,-Courcelle, M., and Schneider, G., 1999, Bifurcating fronts for the Taylor-Couette problem in infinite cylinders. Zeitschrift für Mathematik und Physik, 50: 120-151.

Henry, D., 1981, Geometric Theory of Semilinear Parabolic Equations (Berlin: Springer-Verlag).

Iooss, G., Mielke, A., and Demay, Y., 1989, Theory of steady Ginzburg-Landau equation, in hydrodynamic stability problems. European Journal of Mechanics B/Fluids, 8: 229-268.

Kapitula, T., and Sandstede, B., 1998, Stability of bright solitary wave solutions to perturbed nonlinear Schrödinger equations. Physica D, 124: 58-103.

Lord, G. J., Peterhof, D., Sandstede, B., and Scheel, A., 2000, Numerical computation of solitary waves in infinite cylindrical domains. SIAM Journal of Numerical Analysis, 37: 1420-1454.

Mazin, W., Rasmussen, K. E., Mosekilde, E., Borckmans, P., and Dewel, G., 1996, Pattern formation in the bistable Gray-Scott model. Mathematical Computational Simulations, 40: 371-396.

Palmer, K. J., 1988, Exponential dichotomies and Fredholm operators. Proceedings of the American Mathematical Society, 104: 149-156.

Pego, R., and Weinstein, M., 1994, Asymptotic stability of solitary waves. Communications in Mathematical Physics, 164: 305-349.

Peterhof, D., Sandstede, B., and Scheel, A., 1997, Exponential dichotomies for solitary-wave solutions of semilinear elliptic equations on infinite cylinders. Journal of Differential Equations, 140: 266-308.

Pomeau, Y., 1986, Front motion, metastability and subcritical bifurcations in hydrodynamics. Physica D, 23: $3-11$.

Pospiech, G., 1992, Eigenschaften, Existenz und Stabilität von travelling wave Lösungen zu einem System von Reaktions-Diffusionsgleichungen. PhD thesis, University of Heidelberg.

Sandstede, B., 1998, Stability of multiple-pulse solutions. Transactions of the American Mathematical Society, 350: 429-472.

Sandstede, B., and Scheel, A., 1999a, Essential instability of pulses and bifurcations to modulated travelling waves. Proceedings of the Royal Society of Edinburgh A, 129: 1263-1290.

Sandstede, B., and Scheel, A., 1999b, Absolute and convective instabilities of waves on unbounded and large bounded domains. Physica D, 145: 233-277.

Sandstede, B., and Scheel, A., 1999c, On the structure of spectra of modulated travelling waves. Mathematische Nachrichten (to appear).

Sandstede, B., and Scheel, A., 2000, Spectral stability of modulated travelling waves bifurcating near essential instabilities. Proceedings of the Royal Society of Edinburgh A, 130: 419-448.

Sandstede, B., Scheel, A., and Wulff, C., 1997, Dynamics of spiral waves on unbounded domains using center-manifold reductions. Journal of Differential Equations, 141: 122-149.

Sattinger, D. H., 1976, On the stability of waves of nonlinear parabolic systems. Advanced Mathematics, 22: 312-355.

Schneider, G., 1998, Nonlinear diffusive stability of spatially periodic solutions - abstract theorem and higher space dimensions. Tohoku Math. Publ., 8: 159-167.

Sherratt, J. A., 1998, Invading wave fronts and their oscillatory wakes linked by a modulated travelling phase resetting wave. Physica D, 117: 145-166. 